LA W RENCE LIVERM ORE NATIONAL LABORATORY

Multiple Scattering Correction for Liquid Scintillator Array

J. M. Verbeke, N. J. Snyderman

November 29, 2011 
This document was prepared as an account of work sponsored by an agency of the United States government. Neither the United States government nor Lawrence Livermore National Security, LLC, nor any of their employees makes any warranty, expressed or implied, or assumes any legal liability or responsibility for the accuracy, completeness, or usefulness of any information, apparatus, product, or process disclosed, or represents that its use would not infringe privately owned rights. Reference herein to any specific commercial product, process, or service by trade name, trademark, manufacturer, or otherwise does not necessarily constitute or imply its endorsement, recommendation, or favoring by the United States government or Lawrence Livermore National Security, LLC. The views and opinions of authors expressed herein do not necessarily state or reflect those of the United States government or Lawrence Livermore National Security, LLC, and shall not be used for advertising or product endorsement purposes.

This work performed under the auspices of the U.S. Department of Energy by Lawrence Livermore National Laboratory under Contract DE-AC52-07NA27344. 
UCRL-TR-516871

\title{
Multiple Scattering Correction for Liquid Scintillator Array
}

\author{
Jerome M. Verbeke, N.J. Snyderman \\ Lawrence Livermore National Laboratory
}

October 3, 2009

\section{Introduction}

In this report, we are primarily studying the effect of fast neutrons scattering between different liquid scintillator cells. The reason why this study is important is that if a neutron scatters and deposits enough energy in multiple liquid scintillator cells to record multiple counts, it will not only artificially increase the count rate but also the numbers of two- and three-neutron correlations. It is well known that the masses of nuclear materials undergoing fissions can be determined using ${ }^{3} \mathrm{He}$ tubes measuring thermal neutrons. The method used is based on moments of the random time gate count distributions, which are very sensitive to two-, three- and higher order correlations. Because fast neutrons can scatter multiple times between detectors and register counts artificially correlated, this moment method unfortunately fails for them. Using modified expressions for the moment method to theoretically account for multiple scattering, we will show that the calculated fractions of neutrons scattering multiple times can be used to apply corrections to the masses of nuclear materials undergoing fission.

We propose a method to measure the fractions of neutrons multiple scattering from measuring the spectrum of energies deposited by fast neutrons.

Secondarily, this report also shows that the spectrum of energy deposited by fast neutrons reveals information about the energy spectrum of the neutrons emitted by the source under investigation.

\section{Theory}

When a multipling object is placed in the center of a liquid scintillator array such as the one shown in Fig. 1, one can experimentally measure the times of arrival of the neutrons in each of the liquid scintillator cells. Randomly splitting the sequence of time tags into $N$ segments of length $T$ - where $T$ is of the order of nanoseconds to hundreds of microseconds - one can count how many neutrons arrive in the first segment, how many in the second segment, in the third one, etc. allows one to build a distribution $b_{n}(T)$ of the number $n$ of neutrons arriving in the segments of length $T$. By repeating this procedure for segments of different lengths $T$, multiple count distributions $b_{n}(T)$ can be obtained.

These count distributions $b_{n}(T)$ can be used to determine the strength in units of spontaneous fissions per second $F_{S}$ of the spontaneous fission sources in the object, the efficiency $\varepsilon$ of the liquid scintillator array and

\footnotetext{
*Contact info: verbeke2@1lnl.gov, 925-422-8337
} 


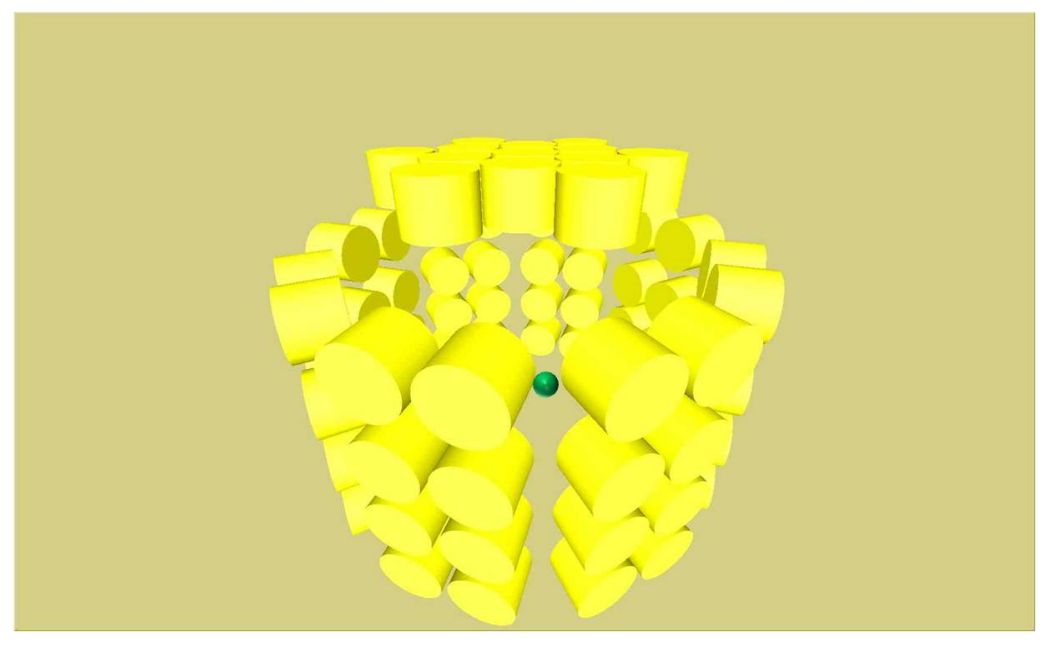

Figure 1: Multiplying object in the middle of a 77 liquid scintillator array.

the multiplication $M$ of the multiplying object. This will be shown by way of the following three equations. One can show theoretically that the first moment of the count distribution $b_{n}(T)$ can be written as

$$
\bar{C}(T)=\varepsilon q(M) M \bar{v}_{s p} F_{s}(1+\alpha) T
$$

where $F_{S}$ is the strength of the spontaneous fission source, $\alpha$ is the ratio of neutrons emitted by sources emitting single neutrons to neutrons emitted by sources emitting multiple neutrons simultaneously, and $q(M) M$ is usually referred to as the escape multiplication and is given by

$$
q(M) M=M-(M-1) / \bar{v}
$$

The symbols $\bar{v}$ and $\bar{v}_{s p}$ are the average numbers of neutrons emitted per induced and spontaneous fissions, respectively. They can be written as

$$
\bar{v}=\sum_{n=1}^{8} n C_{n}
$$

and

$$
\bar{v}_{s p}=\sum_{n=1}^{8} n C_{n}^{s p}
$$

where $C_{n}$ and $C_{n}^{s p}$ are the probabilities of emitting $n$ neutrons per induced and spontaneous fissions, respectively. The upper limit of 8 on the summation sign is the largest number of neutrons that known isotopes produce per fission. In other words, $C_{n}$ is zero for $n$ greater than 8 . It should be noted that the distribution $C_{n}$ depends on the energy of the neutron inducing fission.

The second moment of $b_{n}(T)$ is

$$
Y_{2 F}(T)=\varepsilon q(M) M\left[\frac{D_{2 s}}{1+\alpha}+(M-1) D_{2}\right]\left(1-\frac{1-e^{-\lambda T}}{\lambda T}\right)
$$


where $\lambda$ is a time constant related to the transport of the neutrons in both the measured object and the detection system. $D_{2 s}$ and $D_{2}$ are combinatorial moments of spontaneous and induced fission neutron number distributions. They depend on nuclear data, and are given by

$$
D_{2 s}=\frac{\sum_{n=2}^{8}\left(\begin{array}{l}
n \\
2
\end{array}\right) C_{n}^{s p}}{\bar{v}_{s p}}
$$

and

$$
D_{2}=\frac{\sum_{n=2}^{8}\left(\begin{array}{l}
n \\
2
\end{array}\right) C_{n}}{\bar{v}}
$$

Fig. 2 shows an example of $Y_{2 F}(T)$. The time constant $\lambda^{-1}$ is $6 \mathrm{~ns}$ and the asymptotical value is 0.1174 .
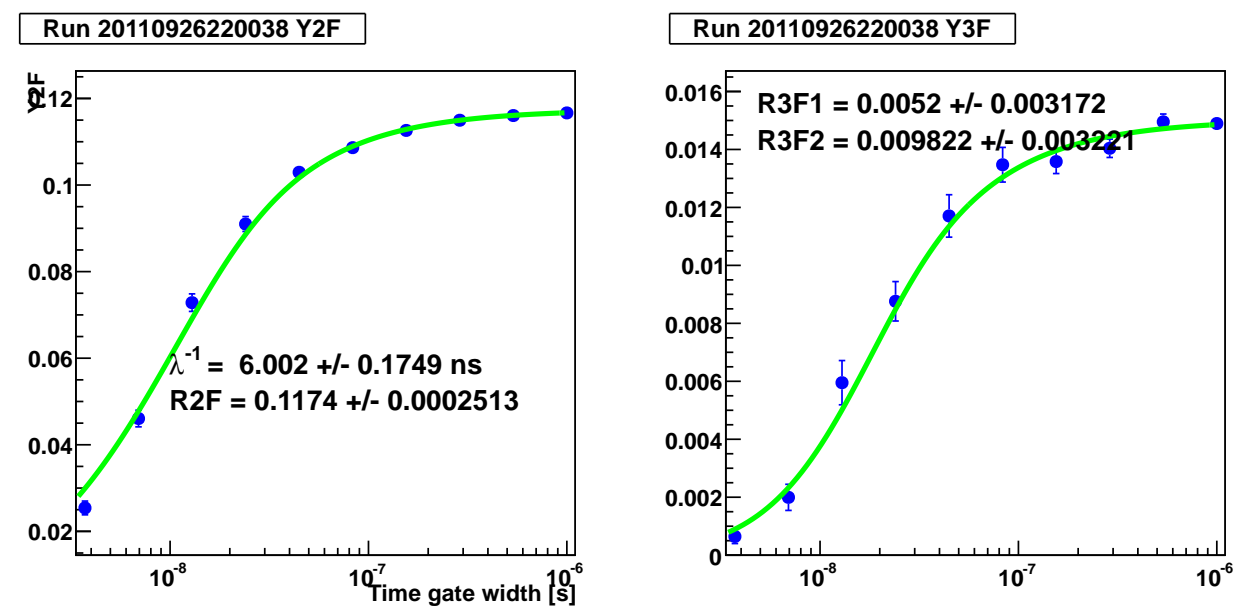

Figure 2: Examples of $Y_{2 F}(T)$ and $Y_{3 F}(T)$.

The third moment of the count distribution $b_{n}(T)$ is

$\begin{aligned} Y_{3 F} & =(\varepsilon q(M) M)^{2} \\ & {\left[\left[\frac{D_{3 s}}{1+\alpha}+(M-1) D_{3}\right]\left(1-\frac{3-4 e^{-\lambda T}+e^{-2 \lambda T}}{2 \lambda T}\right)+\left[2(M-1) \frac{D_{2 s} D_{2}}{1+\alpha}+2(M-1)^{2} D_{2}^{2}\right]\left(1-\frac{2-(2+\lambda T) e^{-\lambda T}}{\lambda T}\right)\right] }\end{aligned}$

where $D_{3 s}$ and $D_{3}$ are combinatorial moments of induced and spontaneous fission neutron number distibutions, and are given by

$$
D_{3 s}=\frac{\sum_{n=3}^{8}\left(\begin{array}{l}
n \\
3
\end{array}\right) C_{n}^{s p}}{\bar{v}_{s p}}
$$

and

$$
D_{3}=\frac{\sum_{n=3}^{8}\left(\begin{array}{l}
n \\
3
\end{array}\right) C_{n}}{\bar{v}}
$$


An example of $Y_{3 F}(T)$ is shown in Fig. 2. The two values $R_{3 F_{1}}$ and $R_{3 F_{2}}$ are the two components of Eq. 8:

$$
\begin{aligned}
& R_{3 F_{1}}=\frac{(\varepsilon q(M) M)^{2}}{1+f_{\text {ext }}}\left[\frac{D_{3 s}}{1+\alpha}+(M-1) D_{3}\right] \\
& R_{3 F_{2}}=\frac{(\varepsilon q(M) M)^{2}}{1+f_{\text {ext }}}\left[2(M-1) \frac{D_{2 s} D_{2}}{1+\alpha}+2(M-1)^{2} D_{2}^{2}\right]
\end{aligned}
$$

so that Eq. 8 can be rewritten as

$$
Y_{3 F}=R_{3 F_{1}}\left(1-\frac{3-4 e^{-\lambda T}+e^{-2 \lambda T}}{2 \lambda T}\right)+R_{3 F_{2}}\left(1-\frac{2-(2+\lambda T) e^{-\lambda T}}{\lambda T}\right)
$$

The slope of Eq. 1 and the asymptotes of Eqs. 5 and 8 for large time gate widths $T$ can be written as

$$
\left\{\begin{array}{l}
R_{1}=\varepsilon q(M) M \bar{v}_{s p} F_{s}(1+\alpha) \\
R_{2 F}=\varepsilon q(M) M\left[\frac{D_{2 s}}{1+\alpha}+(M-1) D_{2}\right] \\
R_{3 F}=(\varepsilon q(M) M)^{2}\left[\frac{D_{3 s}}{1+\alpha}+(M-1) D_{3}+2(M-1) \frac{D_{2 s} D_{2}}{1+\alpha}+2(M-1)^{2} D_{2}^{2}\right]
\end{array}\right.
$$

This system of 3 equations has 4 unknowns $\varepsilon, M, F_{s}$ and $\alpha$. In the absence of $(\alpha, n)$ sources which emit single neutrons at a time, the ratio $\alpha$ is equal to zero. In this case, the system of equations 14 reduces to

$$
\left\{\begin{array}{l}
R_{1}=\varepsilon q(M) M \bar{v}_{s p} F_{s} \\
R_{2 F}=\varepsilon q(M) M\left[D_{2 s}+(M-1) D_{2}\right] \\
R_{3 F}=(\varepsilon q(M) M)^{2}\left[D_{3 s}+(M-1) D_{3}+2(M-1) D_{2 s} D_{2}+2(M-1)^{2} D_{2}^{2}\right]
\end{array}\right.
$$

\subsection{Detector corrections}

These equations rely on the assumption that each neutron in the system can be detected only once. This is certainly true for a detection system based on ${ }^{3} \mathrm{He}$ tubes, because ${ }^{3} \mathrm{He}$ captures the neutron and the neutron disappears from the system. It is different in an array of liquid scintillator cells, because neutrons in such a system deposit energy in cells by scattering with the atoms, and are not absorbed in these elastic and inelastic scattering collisions. They keep traveling, and if they still have enough energy, they can potentially deposit this energy in adjacent or even remote cells. If a neutron scatters multiple times between liquid scintillator cells, Eqs. 1, 5 and 8 no longer hold. They can however be replaced by the count rate $\bar{C}(T)$ and the moments $Y_{2 F}(T)$ and $Y_{3 F}(T)$ that account for the multiple scattering of neutrons (the derivation of these equations is in appendix A, where $\alpha_{2}$ and $\alpha_{3}$ denote $f_{2}$ and $f_{3}$ ):

$$
\left\{\begin{aligned}
\bar{C}(T)= & {\left[\left(1-f_{2}-f_{3}\right)+\left(\begin{array}{l}
2 \\
1
\end{array}\right) f_{2}+\left(\begin{array}{l}
3 \\
1
\end{array}\right) f_{3}\right] R_{1}^{*} T } \\
Y_{2 F}(T) & =\frac{f_{2}+3 f_{3}}{1+f_{2}+2 f_{3}}+\left(1+f_{2}+2 f_{3}\right) \varepsilon q(M) M\left[\frac{D_{2 s}}{1+\alpha}+(M-1) D_{2}\right]\left(1-\frac{1-e^{-\lambda T}}{\lambda T}\right) \\
Y_{3 F}(T) & =\frac{f_{3}}{1+f_{2}+2 f_{3}}+2\left(f_{2}+3 f_{3}\right) \varepsilon q(M) M\left[\frac{D_{2 s}}{1+\alpha}+(M-1) D_{2}\right]\left(1-\frac{1-e^{-\lambda T}}{\lambda T}\right) \\
& +\left(1+f_{2}+2 f_{3}\right)^{2}(\varepsilon q(M) M)^{2} \\
& {\left[\left[\frac{D_{3 s}}{1+\alpha}+(M-1) D_{3}\right]\left(1-\frac{3-4 e^{-\lambda T}+e^{-2 \lambda T}}{2 \lambda T}\right)+\left[2(M-1) \frac{D_{2 s} D_{2}}{1+\alpha}+2(M-1)^{2} D_{2}^{2}\right]\left[1-\frac{2-(2+\lambda T) e^{-\lambda T}}{\lambda T}\right]\right] }
\end{aligned}\right.
$$


where $R_{1}^{*}$ is the true count rate. As opposed to the measured count rate $R_{1}$ which includes 2 counts instead of 1 for each double scatter and 3 counts instead of 1 for each triple scatter, $R_{1}^{*}$ is the rate of individual neutrons detected by the liquid scintillator array. In other words, if a neutron multiple scatters between different liquid scintillator cells, this neutron contributes only a single count towards $R_{1}^{*} . f_{2}$ is the ratio of the doubles rate to the true count rate $R_{1}^{*}, f_{3}$ is the ratio of the triples rate to the true count rate $R_{1}^{*}$. For multiple scatterring, $R_{1}$ is defined as

$$
R_{1}=\left(1+f_{2}+2 f_{3}\right) R_{1}^{*} T
$$

The first equation for $\bar{C}(T)$ in the system of equations 16 is the sum of three terms, all factors of $R_{1}^{*} T$. The first term is the fraction of single neutrons that are counted as such, the second term is $\left(\begin{array}{l}2 \\ 1\end{array}\right)$ times the fraction of single neutrons that are counted twice, the third term is $\left(\begin{array}{l}3 \\ 1\end{array}\right)$ times the fraction of single neutrons that are counted thrice. As a matter of consistency, let us assume the case where single neutrons always double scatter but never triple scatter. In this case $f_{2}$ is 1 and $f_{3}$ is 0 , so that the number of counted neutrons within a time gate $T$ is $\bar{C}(T)=\left(\begin{array}{l}2 \\ 1\end{array}\right) R_{1}^{*} T$, which is twice the number of real single neutrons. The same could be said for the case of neutrons always triple scattering. Equations 16 assume that not a single neutron registers counts in more than 3 liquid scintillator cells. For a single neutron to deposit more than $1.2 \mathrm{MeV}$ - which is approximately the liquid scintillator threshold energy for detecting fast neutrons - in 4 different liquid scintillator cells, it would theoretically only need to have an initial energy of $4.8 \mathrm{MeV}$. In reality however, a neutron would need to have a much higher energy to record counts in 4 different liquid scintillator cells with a reasonable probability. In Fig. 3, we show that the probably of a neutron registering 4 counts in 4 different liquid scintillator cells is negligible for any neutron below $10 \mathrm{MeV}$. Since the purpose here is

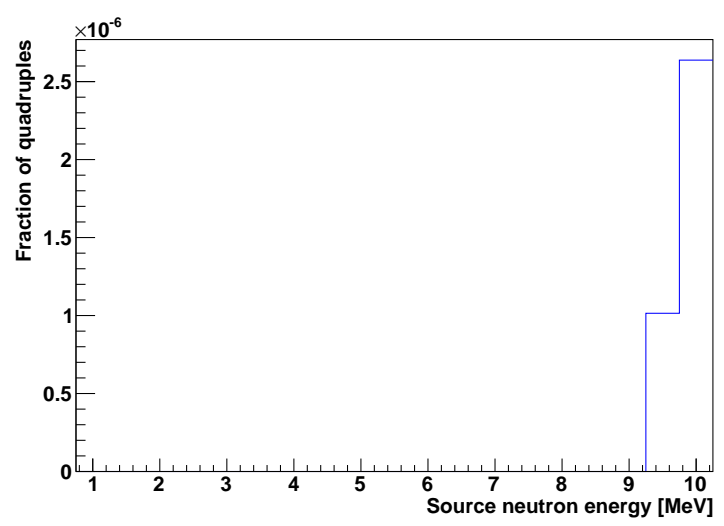

Figure 3: Fraction of detected neutrons registering four counts in the liquid scintillator cells, as a function of the source neutron energies.

to study fast neutrons with energies similar to the ones found in fission spectra, we can safely neglect any multiple scattering greater than 3.

Once we know $f_{2}$ and $f_{3}$, and if we assume that $\alpha$ is zero, the system of equations 16 corrected for multiple scattering can be solved for the three system parameters: the strength $F_{S}$ of the spontaneous fission source, the multiplication $M$ of the object, and the efficiency $\varepsilon$ of the detection system. Our goal is to determine whether the multiple scattering fractions $f_{2}$ and $f_{3}$ can be determined from measuring the spectrum of 
energies deposited by the fast neutrons from a single measurement, that is without any prior measurement. If this were the case, we could easily use these fractions to determine the system parameters. 


\section{Neutron energy sensitivity study}

For this study, we considered the liquid scintillator array shown in Fig. 1. We assumed a point neutron source is located in the middle of the array of liquid scintillators depicted in Fig. 1. Neutrons are emitted one at a time from that point source. For 19 different mono-energetic neutron beams ranging from $1 \mathrm{MeV}$ to $10 \mathrm{MeV}$ in increments of $0.5 \mathrm{MeV}$, we use the Monte Carlo radiation transport code MCNPX to transport the neutrons from the point source in the middle of the array through the geometry consisting of the array of liquid scintillator cells.

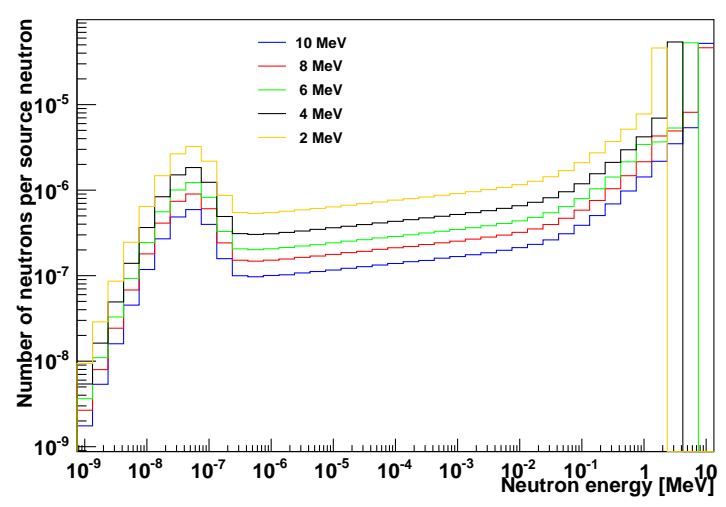

Figure 4: Distributions of numbers of fast neutrons traversing the liquid scintillator cells as predicted by MCNPX. The different colors refer to a subset of the initial neutron energies.

It is interesting to first take a peek at the ensemble of the energies of the neutrons as they traverse the liquid scintillator cells, shown in Fig. 4. One notices than most neutrons have their full energy as they traverse the liquid scintillator cells, but there is also a second relatively strong peak from the neutrons which have been thermalized by the hydrogen in the liquid scintillator cells themselves. The spectrum of energies
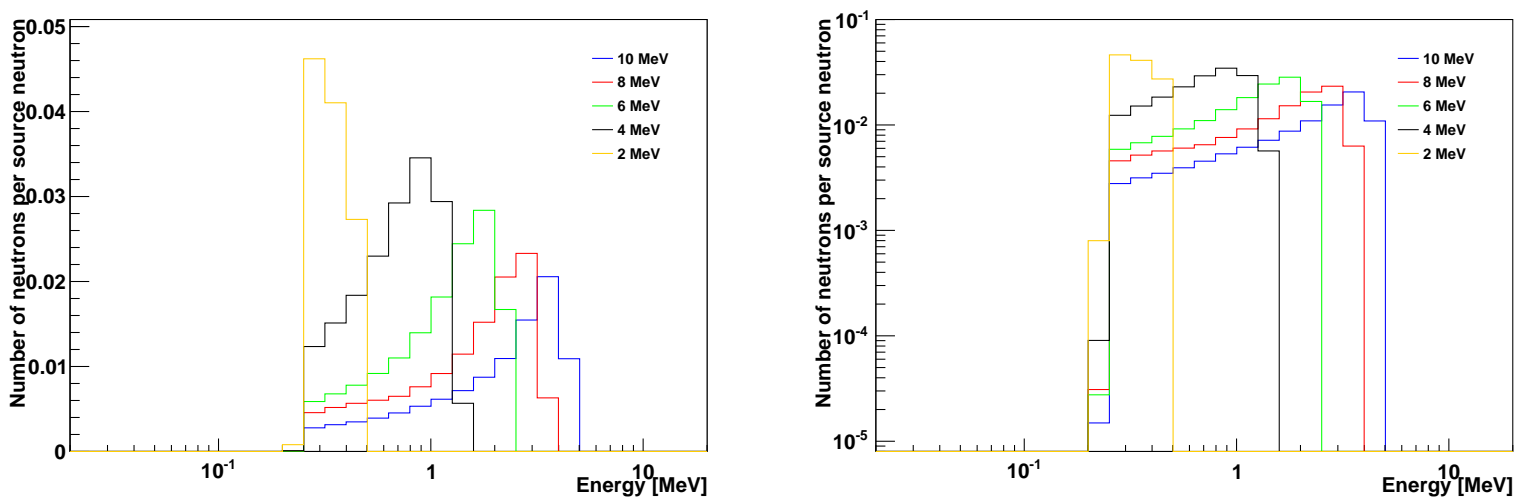

Figure 5: Distributions of numbers of fast neutrons as a function of the energy deposited in the liquid scintillator cells as predicted by MCNPX. The different colors refer to a subset of the initial neutron energies. 
deposited by fast neutrons is shown in Fig. 5, where multiple scattering of neutrons is included. This figure shows that source neutrons with different energies produce different responses, that is different spectra of deposited energies in the liquid scintillator cells.

The last set of figures 6 shows the probability density functions for the energies deposited by the neutrons in the liquid scintillator cells. To produce these distributions, each distribution in Fig. 5 was normalized, and each bin of the normalized distribution was divided by the bin width. Integrated over the energy range, each cumulative density function thus produced is equal to 1 .
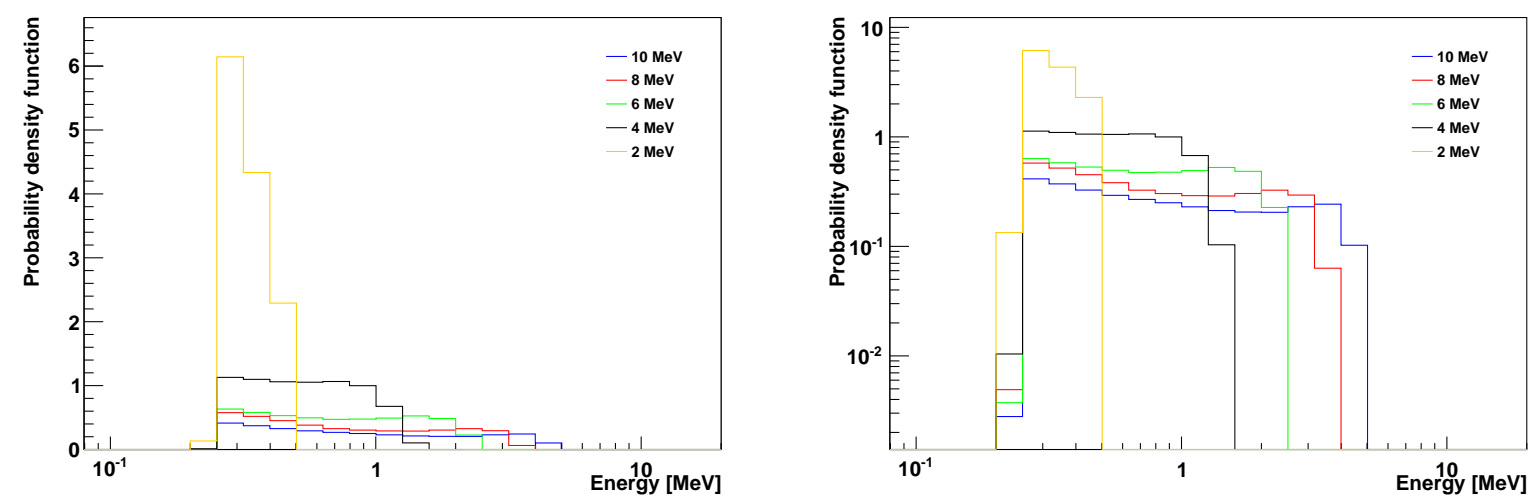

Figure 6: Probability density functions for the energies deposited by mono-energetic source neutrons in the liquid scintillator cells as predicted by MCNPX. The different colors refer to a subset of the initial neutron energies.

\subsection{First method to determine multiple scattering fractions}

For these 19 energies between $1 \mathrm{MeV}$ and $10 \mathrm{MeV}$, we computed the fractions of single source neutrons registering counts in two and three liquid scintillator cells. The simulations were such that (a) the system does not multiply neutrons, so that both $M$ and $q(M) M$ are equal to 1, and (b) neutrons are emitted one at a time, so that both $D_{2 s}$ and $D_{3 s}$ are zero. In these conditions, many terms disappear in the system of equations 16, and we can write the following equations that apply for non-multiplying sources emitting single neutrons at a time:

$$
\left\{\begin{array}{l}
\bar{C}(T)=\left(1+f_{2}+2 f_{3}\right) R_{1}^{*} T \\
Y_{2}(T)=\left(f_{2}+3 f_{3}\right) R_{1}^{*} T \\
Y_{3}(T)=f_{3} R_{1}^{*} T
\end{array}\right.
$$

where $Y_{2}(T)$ and $Y_{3}(T)$ are equal to $Y_{2 F}(T)$ and $Y_{3 F}(T)$ multiplied by $\bar{C}(T)$. This system of 3 equations has 3 unknowns. The fractions of doubles $f_{2}$ and triples $f_{3}$ as well as the true count rate $R_{1}^{*}$ can be determined by taking the slopes of Eqs. 18.

These equations are better illustrated by looking at the quantities $\bar{C}(T), Y_{2}(T)$, and $Y_{3}(T)$ for a simulation of a weak $8 \mathrm{MeV}$-neutron source in the middle of the 77-cell liquid scintillator array. Fig. 7 shows 4 quadrants: the top left shows the count distribution of fast neutrons for a time gate of width $512 \mathrm{~ns}$, the top right is $\bar{C}(T)$, the bottom left and right are $Y_{2}(T)$ and $Y_{3}(T)$, respectively. The values for the moments 
come from 512 count distributions similar to the one shown in the top left quadrant of Fig. 7 for time gate widths ranging from 16 to $512 \mathrm{~ns}$. Blue is Monte-Carlo data, green is theoretical fits. The slopes of $\bar{C}(T)$ in the top right quadrant, of $Y_{2}(T)$ and $Y_{3}(T)$ in the bottom quadrants of Fig. 7 are the factors of $T$ in Eqs. 18.

Given the size of the liquid scintillator cells (10-cm-diameter), and the speed of $\sim 1.5 \mathrm{~cm} / \mathrm{ns}$ of $1 \mathrm{MeV}$ neutrons, it would take a neutron at least $7 \mathrm{~ns}$ to register a count in a cell, register a count in a second cell and travel $10 \mathrm{~cm}$ to get to a third cell. Even for $4 \mathrm{MeV}$ neutrons traveling twice as fast, it would take $3.5 \mathrm{~ns}$ for them to register counts in 3 different cells. From this follows that the slope of $Y_{3}(T)$ is not quite as steep when $\mathrm{T}$ is close to 0 . The same can be said for $Y_{2}(T)$. Because of this unmodeled latency - even though short - the moments $Y_{2}(T)$ and $Y_{3}(T)$ in Fig. 7 are not fit by the linear functions 18, but by affine versions of them with identical slopes:

$$
\left\{\begin{array}{l}
\bar{C}(T)=\left(1+f_{2}+2 f_{3}\right) R_{1}^{*} T \\
Y_{2}(T)=\left(f_{2}+3 f_{3}\right) R_{1}^{*}\left(T-T_{2}^{o}\right) \\
Y_{3}(T)=f_{3} R_{1}^{*}\left(T-T_{3}^{o}\right)
\end{array}\right.
$$

The values of $f_{2}, f_{3}$ and $R_{1}^{*}$ can be determined by solving the system of equations 19 . Interestingly, $T_{2}^{o}$ and $T_{3}^{o}$ give orders of magnitude for the times it takes for neutrons to register 2 and 3 liquid scintillator counts, respectively. Fig. 7 reveals that $8-\mathrm{MeV}$ neutrons take in average $\sim 6.6 \mathrm{~ns}$ to scatter from one liquid scintillator cell to another one, while they take in average $\sim 12 \mathrm{~ns}$ to scatter from one liquid scintillator cell to two other ones, consecutively. The reason why triple-scattering does not take twice as long as doublescattering can be explained. When a neutron scatters and records counts in 2 cells, the energy of the neutron between the first and second cells can be as low as $1.2 \mathrm{MeV}$ (threshold for detection of fast neutrons). For a neutron to scatter and record counts in 3 cells, the energy of the neutron between the first and second cells has to be at least $2.4 \mathrm{MeV}$. Otherwise, the fast neutron - which needs to deposit at least $1.2 \mathrm{MeV}$ in the second cell to be counted, and would thus be left with at the most $1.2 \mathrm{MeV}$ after the second cell — would not have the required $1.2 \mathrm{MeV}$ of energy to record a count in a third cell. Therefore, for three counts to be recorded, the speed of the neutron between the first and the second cell has to be at least 1.4 times greater than the speed of a neutron recording only two counts. Thus, we can conclude that the time it takes for a neutron to record 3 counts in a liquid scintillator array will be less than twice the time it takes for a neutron to record 2 counts in the same liquid scintillator array. 

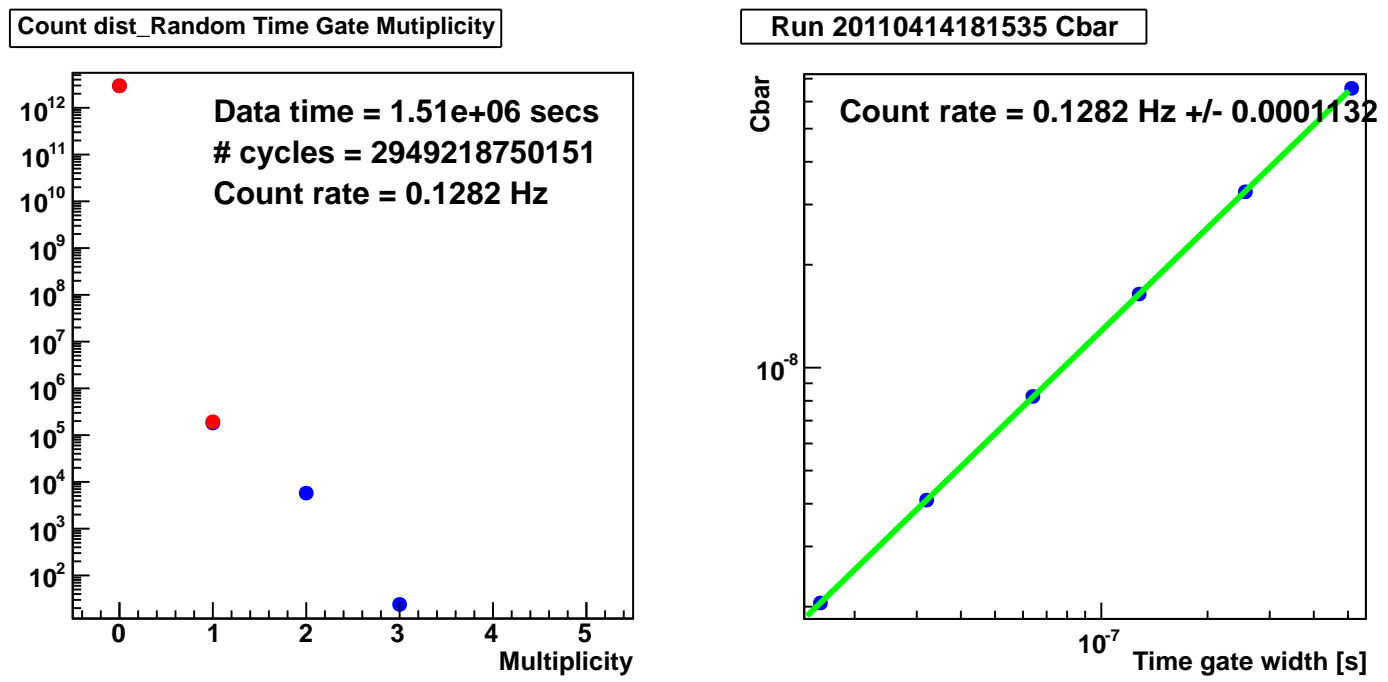

\section{Run 20110414181535 Y2}
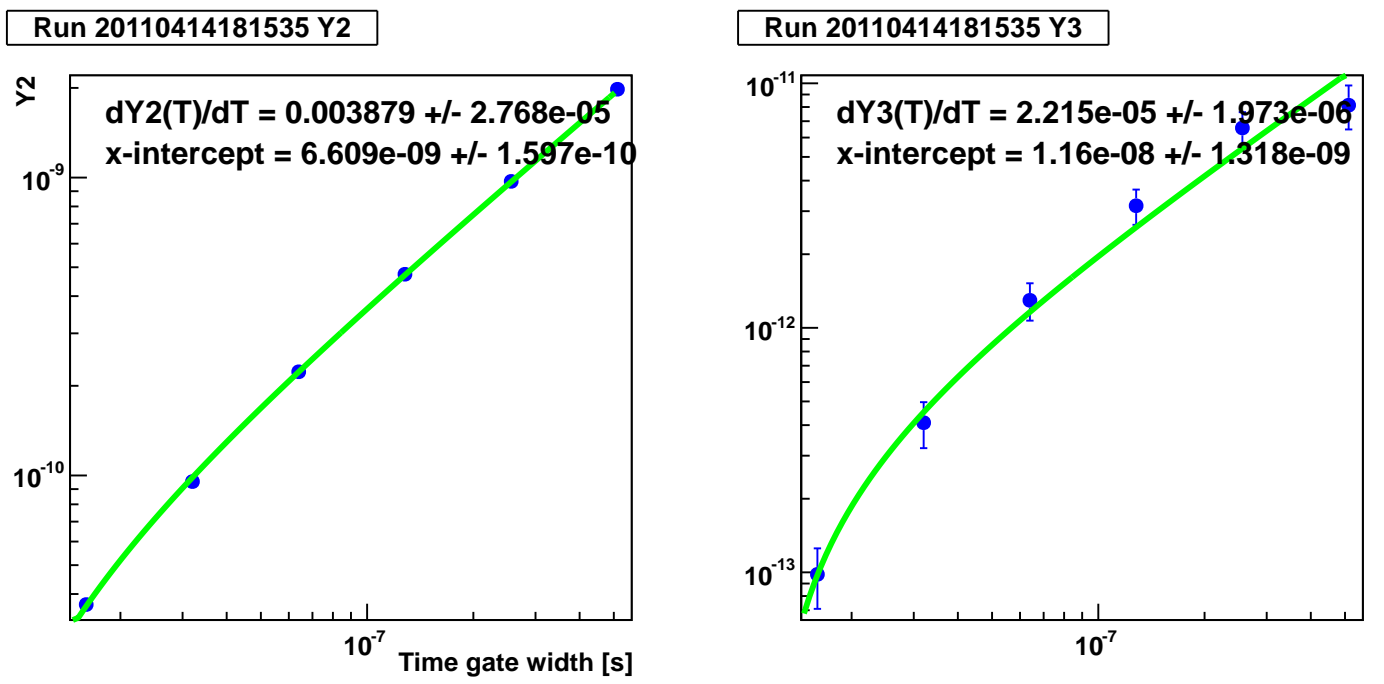

Figure 7: Count distribution $b_{n}(\mathrm{~T}=512 \mathrm{~ns})$ and first three cumulant moments $\bar{C}(T), Y_{2}(T)$ and $Y_{3}(T)$ as a function of time gate width $T$, for $T$ ranging between $16 \mathrm{~ns}$ and $512 \mathrm{~ns}$. The cumulant moments are fit using Eqs. 19. The data are from a MCNPX simulation of $8 \mathrm{MeV}$ neutrons in the middle of the liquid scintillator array shown in Fig. 1. Blue is Monte Carlo data, green is theoretical fit. 
The fractions $f_{2}$ and $f_{3}$ of neutrons scattering multiple times and registering two and three counts in the liquid scintillator cells are shown in Fig. 8 for a range of source neutron energies. The fraction of neutrons
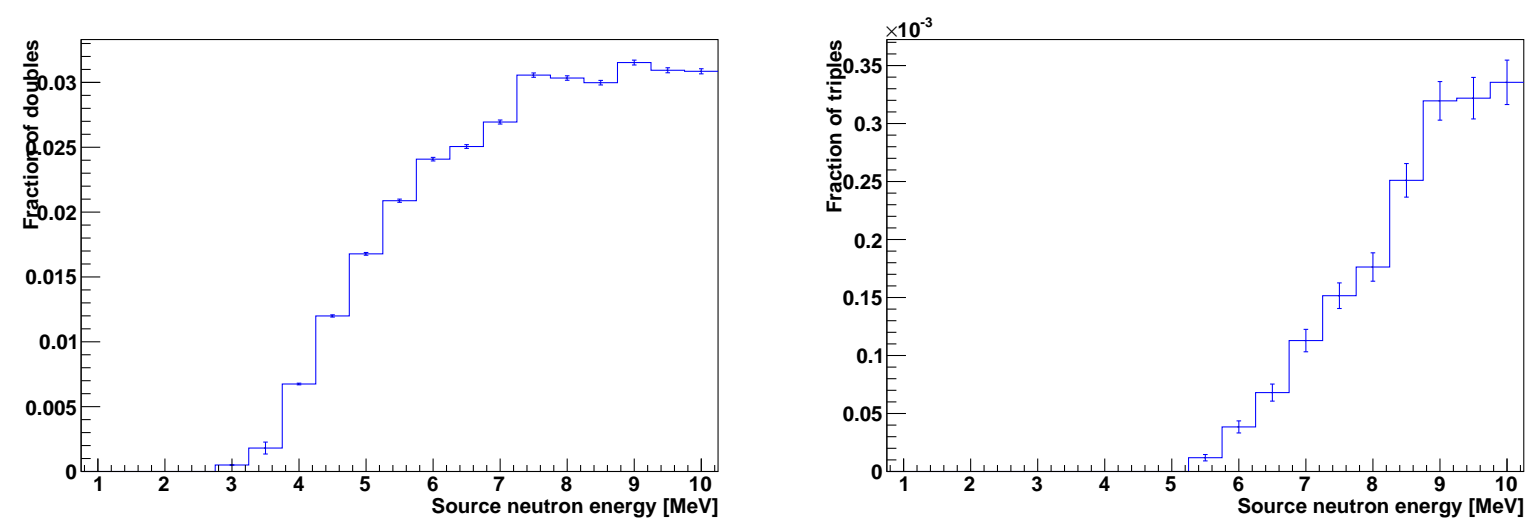

Figure 8: Fractions $f_{2}$ and $f_{3}$ of detected neutrons registering two and three counts in the liquid scintillator cells, as a function of the source neutron energies.

scattering multiple times and registering four counts in the liquid scintillator cells is shown in Fig. 3.

Because the count rate is artificially inflated by the neutrons registering multiple counts in the liquid scintillator cells, the true count rate differs from the measured count rate as shown in Fig. 9. This figure shows the true count rate $R_{1}^{*}$ and measured count rates $R_{1}$ as a function of the source neutron energies. Note that the 3 plotted count rates are exactly matched up to $3 \mathrm{MeV}$, and are thus hidden behind each other. Furthermore, the "Measured count rate $R_{1}$ " and "Count rate $R_{1}$ determined by method 2" pretty much exactly match all the way to $10 \mathrm{MeV}$.
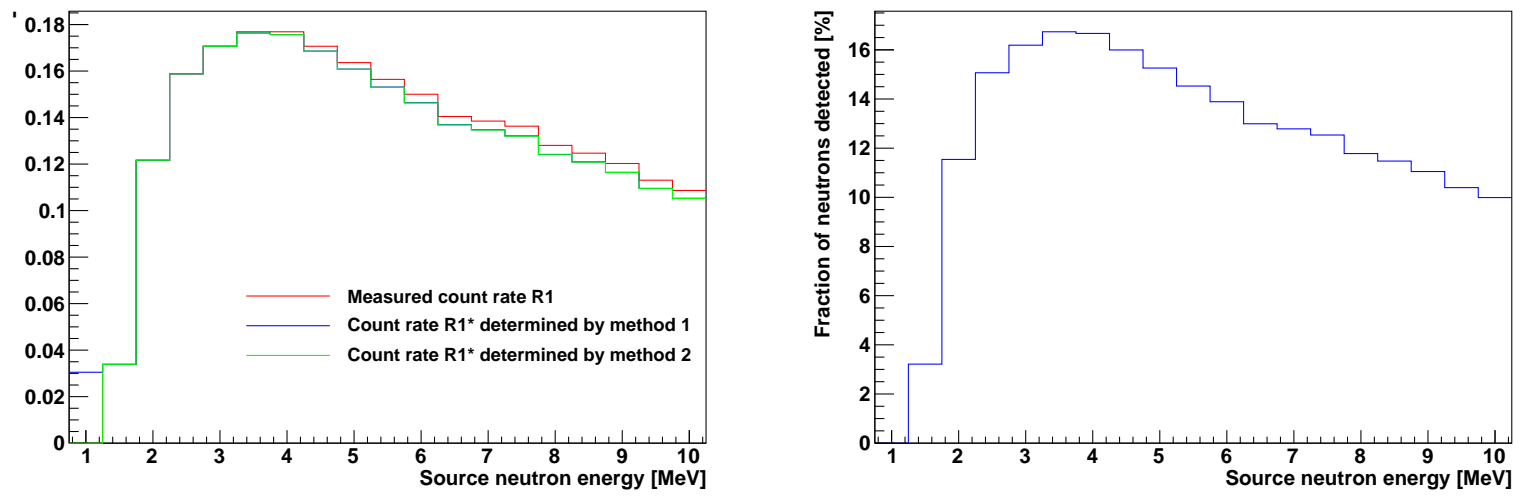

Figure 9: Comparison between the measured count rate $R_{1}$, the count rate $R_{1}^{*}$ predicted by the system of equations 16 and the count rate predicted by the second method, as a function of the different initial neutron energies. Neutron detection efficiency of liquid scintillator array, as a function of neutron energy.

The right graph in Fig. 9 shows the absolute neutron detection efficiency of the liquid scintillator array. The detection efficiency is maximum for neutron energies around $3.5 \mathrm{MeV}$ and $4 \mathrm{MeV}$, and decreases 
steadily as the neutron energy increases. Fast neutrons $1 \mathrm{MeV}$ and lower are undetectable.

The fractions $f_{2}$ and $f_{3}$ of neutrons scattering multiple times are listed in table 1 for different monoenergetic neutron sources, along with $a_{1}, b_{1}, b_{2}, c_{1}, c_{2}$ and $c_{3}$, which are the coefficients that enter into the system of equations 16 .

Table 1: Fractions $f_{2}$ and $f_{3}$ of neutrons double- and triplescattering between liquid scintillator cells, for mono-energetic and fissile neutrons incident on the array shown in Fig. 1. The liquid scintillator energy threshold is $250 \mathrm{keV}$.

\begin{tabular}{|c|c|c|c|c|c|c|c|c|}
\hline Neutron source & $f_{2}[\%]$ & $f_{3}[\%]$ & $a_{1}$ & $b_{1}$ & $b_{2}$ & $c_{1}$ & $c_{2}$ & $c_{3}$ \\
\hline $1.5 \mathrm{MeV}$ & $000 \mathrm{e}+00$ & $0.000 \mathrm{e}+00$ & 1.0000 & $0.000 \mathrm{e}+00$ & 1.0000 & $0.000 \mathrm{e}+00$ & $0.000 \mathrm{e}+00$ & 1.0000 \\
\hline $2.0 \mathrm{MeV}$ & $0.000 \mathrm{e}+00$ & $0.000 \mathrm{e}+00$ & 1.0000 & $0.000 \mathrm{e}+00$ & 1.0000 & $0.000 \mathrm{e}+00$ & $0.000 \mathrm{e}+00$ & 1.0000 \\
\hline $2.5 \mathrm{MeV}$ & $0.000 \mathrm{e}+00$ & $0.000 \mathrm{e}+00$ & 1.0000 & $0.000 \mathrm{e}+00$ & 1.0000 & $0.000 \mathrm{e}+00$ & $0.000 \mathrm{e}+00$ & 1.0000 \\
\hline $3.0 \mathrm{MeV}$ & $5.008 \mathrm{e}-02$ & $0.000 \mathrm{e}+00$ & 1.0005 & $5.005 \mathrm{e}-04$ & 1.0005 & $0.000 \mathrm{e}+00$ & $1.002 \mathrm{e}-03$ & 1.0010 \\
\hline $3.5 \mathrm{MeV}$ & $1.808 \mathrm{e}-01$ & $0.000 \mathrm{e}+00$ & 1.0018 & $804 \mathrm{e}-03$ & 1.0018 & $0.000 \mathrm{e}+00$ & e-03 & 1.0036 \\
\hline $4.0 \mathrm{MeV}$ & $6.746 \mathrm{e}-01$ & $0.000 \mathrm{e}+00$ & 1.0067 & $6.701 \mathrm{e}-03$ & 1.0067 & $0.000 \mathrm{e}+00$ & $1.349 \mathrm{e}-02$ & 1.0135 \\
\hline $4.5 \mathrm{MeV}$ & $1.199 \mathrm{e}+00$ & $0.000 \mathrm{e}+00$ & 1.0120 & $1.185 \mathrm{e}-02$ & 1.0120 & $0.000 \mathrm{e}+00$ & $2.399 \mathrm{e}-02$ & 1.0241 \\
\hline $5.0 \mathrm{MeV}$ & $1.678 \mathrm{e}+00$ & $0.000 \mathrm{e}+00$ & 1.0168 & e- 02 & 1.0168 & +00 & e-02 & 1.0338 \\
\hline $5.5 \mathrm{MeV}$ & $2.088 \mathrm{e}+00$ & $1.188 \mathrm{e}-03$ & 1.0209 & $2.049 \mathrm{e}-02$ & 1.0209 & $4 e-05$ & e-02 & 1.0422 \\
\hline $6.0 \mathrm{MeV}$ & $2.408 \mathrm{e}+00$ & $3.843 \mathrm{e}-03$ & 1.0242 & -02 & 1.0242 & 05 & -02 & 1.0489 \\
\hline $6.5 \mathrm{MeV}$ & $2.506 \mathrm{e}+00$ & $6.802 \mathrm{e}-03$ & 1.0252 & e-02 & 1.0252 & -05 & -02 & 1.0510 \\
\hline 7.0 MeV & $2.695 \mathrm{e}+00$ & $1.129 \mathrm{e}-02$ & 1.0272 & $2.656 \mathrm{e}-02$ & 1.0272 & $1.099 \mathrm{e}-04$ & $5.457 \mathrm{e}-02$ & 1.0551 \\
\hline $7.5 \mathrm{MeV}$ & $3.056 \mathrm{e}+00$ & $1.516 \mathrm{e}-02$ & 1.0309 & $3.009 \mathrm{e}-02$ & 1.0309 & e- 04 & e- 02 & 1.0627 \\
\hline $8.0 \mathrm{MeV}$ & $3.034 \mathrm{e}+00$ & $1.763 \mathrm{e}-02$ & 1.0307 & $2.995 \mathrm{e}-02$ & 1.0307 & $1.710 \mathrm{e}-04$ & $6.173 \mathrm{e}-02$ & 1.0623 \\
\hline $8.5 \mathrm{MeV}$ & $2.998 \mathrm{e}+00$ & $2.510 \mathrm{e}-02$ & 1.0305 & $2.982 \mathrm{e}-02$ & 1.0305 & $2.436 \mathrm{e}-04$ & e-02 & 1.0619 \\
\hline $9.0 \mathrm{MeV}$ & $3.153 \mathrm{e}+00$ & $3.195 \mathrm{e}-02$ & 1.0322 & $3.148 \mathrm{e}-02$ & 1.0322 & $3.096 \mathrm{e}-04$ & $6.498 \mathrm{e}-02$ & 1.0654 \\
\hline $9.5 \mathrm{MeV}$ & $3.094 \mathrm{e}+00$ & $3.219 \mathrm{e}-02$ & 1.0316 & $3.093 \mathrm{e}-02$ & 1.0316 & $3.120 \mathrm{e}-04$ & $6.380 \mathrm{e}-02$ & 1.0642 \\
\hline $10.0 \mathrm{MeV}$ & $3.086 \mathrm{e}+00$ & $3.355 \mathrm{e}-02$ & 1.0315 & $3.089 \mathrm{e}-02$ & 1.0315 & $3.253 e-04$ & $6.373 \mathrm{e}-02$ & 1.0640 \\
\hline${ }^{238} \mathrm{U}$ & $2.786 \mathrm{e}-01$ & $0.000 \mathrm{e}+00$ & 1.0028 & $2.778 \mathrm{e}-03$ & 1.0028 & $0.000 \mathrm{e}+00$ & $5.572 \mathrm{e}-03$ & 1.0056 \\
\hline${ }^{240} \mathrm{Pu}$ & $3.875 \mathrm{e}-01$ & $3.407 \mathrm{e}-04$ & 1.0039 & $3.870 \mathrm{e}-03$ & 1.0039 & $3.394 \mathrm{e}-06$ & $7.770 \mathrm{e}-03$ & 1.0078 \\
\hline${ }^{252} \mathrm{Cf}$ & $4.245 \mathrm{e}-01$ & $9.739 \mathrm{e}-04$ & 1.0043 & $4.256 \mathrm{e}-03$ & 1.0043 & $9.698 \mathrm{e}-06$ & $8.549 \mathrm{e}-03$ & 1.0085 \\
\hline $2.2 \mathrm{MeV}$ (Fig. 12) & $0.000 \mathrm{e}+00$ & $0.000 \mathrm{e}+00$ & 1.0000 & $0.000 \mathrm{e}+00$ & 1.0000 & $0.000 \mathrm{e}+00$ & $0.000 \mathrm{e}+00$ & 1.0000 \\
\hline 4.4 MeV (Fig. 10) & $1.113 \mathrm{e}+00$ & $0.000 \mathrm{e}+00$ & 1.0111 & $1.101 \mathrm{e}-02$ & 1.0111 & $0.000 \mathrm{e}+00$ & $2.226 \mathrm{e}-02$ & 1.0224 \\
\hline 7.24 MeV (Fig. 11) & $2.680 \mathrm{e}+00$ & $1.307 \mathrm{e}-02$ & 1.0271 & $2.647 \mathrm{e}-02$ & 1.0271 & $1.272 \mathrm{e}-04$ & $5.438 \mathrm{e}-02$ & 1.0549 \\
\hline
\end{tabular}

Their definitions are given in Eqs. 20 through 25.

$$
\begin{aligned}
& a_{1}=1+f_{2}+2 f_{3} \\
& b_{1}=\frac{f_{2}+3 f_{3}}{1+f_{2}+2 f_{3}} \\
& b_{2}=1+f_{2}+2 f_{3} \\
& c_{1}=\frac{f_{3}}{1+f_{2}+2 f_{3}}
\end{aligned}
$$




$$
\begin{aligned}
& c_{2}=2\left(f_{2}+3 f_{3}\right) \\
& c_{3}=\left(1+f_{2}+2 f_{3}\right)^{2}
\end{aligned}
$$

For the sake of clarity, system of equations 16 is rewritten here using the new coefficients:

$$
\left\{\begin{array}{l}
\bar{C}(T)=a_{1} R_{1}^{*} T \\
Y_{2 F}(T)=b_{1}+b_{2} \varepsilon q(M) M\left[\frac{D_{2 s}}{1+\alpha}+(M-1) D_{2}\right]\left(1-\frac{1-e^{-\lambda T}}{\lambda T}\right) \\
Y_{3 F}(T)=c_{1}+c_{2} \varepsilon q(M) M\left[\frac{D_{2 s}}{1+\alpha}+(M-1) D_{2}\right]\left(1-\frac{1-e^{-\lambda T}}{\lambda T}\right)+c_{3}(\varepsilon q(M) M)^{2} \\
\\
{\left[\left[\frac{D_{3 s}}{1+\alpha}+(M-1) D_{3}\right]\left(1-\frac{3-4 e^{-\lambda T}+e^{-2 \lambda T}}{2 \lambda T}\right)+\left[2(M-1) \frac{D_{2 s} D_{2}}{1+\alpha}+2(M-1)^{2} D_{2}^{2}\right]\left[1-\frac{2-(2+\lambda T) e^{-\lambda T}}{\lambda T}\right]\right]}
\end{array}\right.
$$

One should point out that the multiple scattering fractions $f_{2}$ and $f_{3}$ greatly depend on the geometry of the liquid scintillator array and on the energy threshold of the liquid scintillator cells. The values in table 1 are only valid for the geometry shown in Fig. 1 and for a liquid scintillator energy threshold of $250 \mathrm{keV}$. 


\subsection{Second method to determine multiple scattering fractions}

To make sure the analysis using the system of equations 16 was derived properly, we derived the numbers of multiple scatterings not from a statistical perspective, but from an alternative second method. This can be done simply using the procedure described here: each fast neutron count registered by the liquid scintillator cells triggers a time window of length $T$ and one counts how many fast neutrons are within that window. If there is one fast neutron in the window, then this count along with the one that triggered the window will be consolidated into a single double scattering count. If there were two fast neutrons in the window, then these 2 counts along with the trigger count will be consolidated into a single triple scattering count. The empty windows are considered single scattering events, because they only contain the trigger event. The fractions of doubles and triples are quasi-identical to the ones shown in Fig. 8, the count rates do not differ much either, as shown by the "method 2" line in Fig. 9. These two ways ot producing the same data gives us confidence that the models used to predict the unknowns $R_{1}^{*}, f_{2}$ and $f_{3}$ are correct.

\section{Input spectrum reconstruction}

Before trying to determine the factors $f_{2}$ and $f_{3}$, we will see if we can reconstruct the spectra of 3 different mono-energetic neutron sources, based on the set of spectra of deposited energies shown in Fig. 5. We use the subscript $E_{i}$ to distinguish each one of the basis functions $g_{E_{i}}\left(E_{d}\right)$ shown in Fig. 5. The subscript $E_{i}$ denotes the different initial source neutron energies, from $1 \mathrm{MeV}(\mathrm{i}=1)$ up to $10 \mathrm{MeV}$ (i=19), in increments of $0.5 \mathrm{MeV}$. Given a measured spectrum of deposited energies $\tilde{g}\left(E_{d}\right)$, the goal for the reconstruction is to find the set of weights $w_{E_{i}}$ for which the difference between $\tilde{g}\left(E_{d}\right)$ and the reconstructed energy deposition spectrum is minimized. The weights $w_{E_{i}}$ are physically to be interpreted as the strengths of the neutron sources of energy $E_{i}$. If the distribution $\tilde{g}\left(E_{d}\right)$ is normalized, the weights $w_{E_{i}}$ will be the relative strengths of the different neutron sources of energy $E_{i}$.

Let's call $g^{r}\left(E_{d}\right)$ the reconstructed energy deposition spectrum. $g^{r}\left(E_{d}\right)$ is defined as

$$
g^{r}\left(E_{d}\right)=\sum_{i=1}^{19} w_{E_{i}} g_{E_{i}}\left(E_{d}\right)
$$

The optimal set of weights $w_{E_{i}}$ will be such as to minimize

$$
g^{r}\left(E_{d}\right)-\tilde{g}\left(E_{d}\right)
$$

In order to minimize Eq. 28, we use $\chi$-square minimization algorithm implemented by Minuit in ROOT. Based on this optimization, an estimate of the source neutron spectrum will be given by the energies $E_{i}$ weighed by the weights $w_{E_{i}}$. For mono-energetic neutron sources, the estimated source neutron energy $E^{e s t}$ will be given by the $w_{E_{i}}$ weighed average of the $E_{i}$.

$$
E^{e s t}=\sum_{i=1}^{19} w_{E_{i}} E_{i}
$$

Based on the solution set for the weights $w_{E_{i}}$, we can produce the spectrum of energies deposited by the neutrons using

$$
g^{e s t}\left(E_{d}\right)=\sum_{i=1}^{19} w_{E_{i}} g_{E_{i}}\left(E_{d}\right)
$$




\subsection{Reconstruction of mono-energetic neutron sources}

We ran a few simulations similar to the ones in Sec. 3, but with energies not among the $E_{i}$ for which we have basis functions. The first energy of mono-energetic neutrons considered was $4.4 \mathrm{MeV}$. Fig. 10 shows the reconstructed source neutron energy spectrum and with the spectrum of energies deposited by the neutron constructed from the reconstructed source neutron energy spectrum using Eq. 30. Because we know
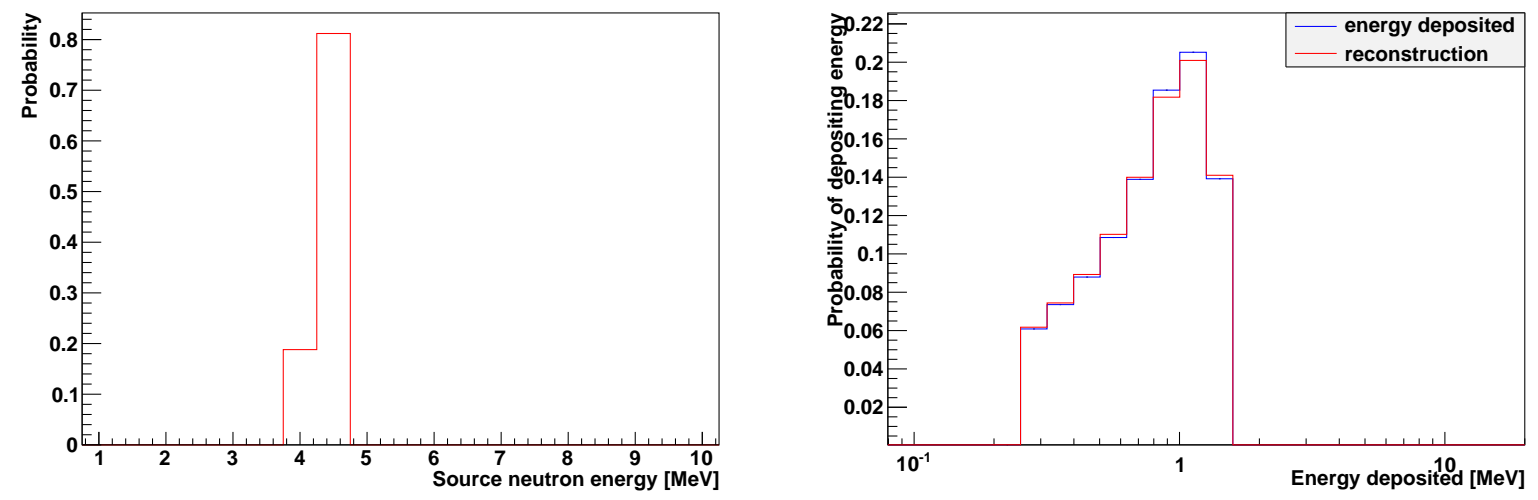

Figure 10: (a) Reconstruction of the source neutron energy spectrum using Eq. 28. (b) Reconstructed spectrum $g^{e s t}\left(E_{d}\right)$ of energies deposited by the neutrons, evaluated from Eq. 30. The estimated average source neutron energy is $4.406 \mathrm{MeV}$. The data is from a MCNPX simulation of a mono-energetic $4.4 \mathrm{MeV}$ neutron source.

the neutron source was mono-energetic, we used Eq. 29 to compute the energy of the neutron source, the reconstructed energy was estimated at $4.406 \mathrm{MeV}$. The reconstruction of the deposited energy spectrum is not perfect but nonetheless a very good approximation of the one "measured" directly in the modeled liquid scintillator cells.

The second and third simulations are mono-energetic 7.24 MeV and 2.2 MeV neutron source in the same liquid scintillator array. The reconstructed source neutron energy spectra and the spectra of energies deposited by the neutron constructed from the reconstructed source neutron energy spectra are shown in Fig. 11 and 12. The average energies determined from the reconstructions were $7.51 \mathrm{MeV}$ and $2.22 \mathrm{MeV}$. From these few data points, we observe that the predictions of the source neutron energies are good, but not perfect. Fig. 11 indeed shows that there is a somewhat unexpected secondary peak at $8.5 \mathrm{MeV}$, while we would have expected a secondary peak at $7.5 \mathrm{MeV}$. 

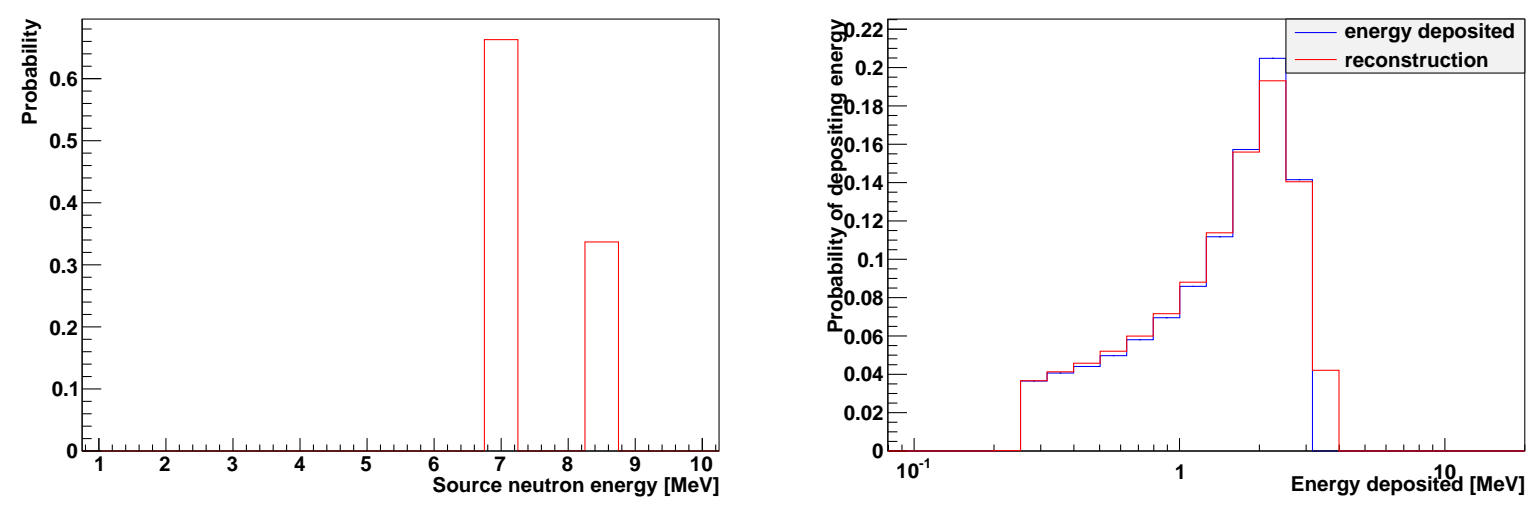

Figure 11: (a) Reconstruction of the source neutron energy spectrum using Eq. 28. (b) Reconstructed spectrum $g^{\text {est }}\left(E_{d}\right)$ of energies deposited by the neutrons, evaluated from Eq. 30. The estimated average source neutron energy is $7.51 \mathrm{MeV}$. The data is from a MCNPX simulation of a mono-energetic $7.24 \mathrm{MeV}$ neutron source.
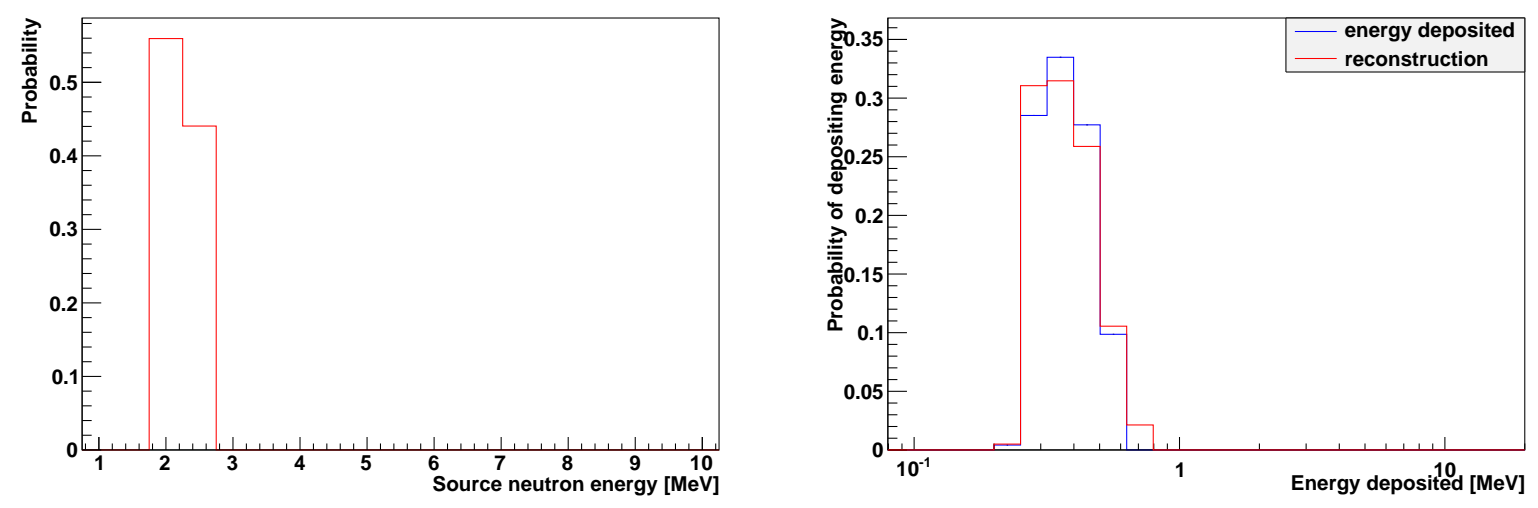

Figure 12: (a) Reconstruction of the source neutron energy spectrum using Eq. 28. (b) Reconstructed spectrum $g^{e s t}\left(E_{d}\right)$ of energies deposited by the neutrons, evaluated from Eq. 30. The estimated average source neutron energy is $2.22 \mathrm{MeV}$. The data is from a MCNPX simulation of a mono-energetic $2.2 \mathrm{MeV}$ neutron source. 


\subsection{Reconstruction of spontaneous fission sources.}

The next test is to see whether the reconstruction algorithm could predict the neutron spectrum emitted by spontaneous fission sources. Of course, the caveat here is one should not expect to predict the source neutron spectrum under approximately $1.25 \mathrm{MeV}$, since liquid scintillators cannot detect neutrons below that energy.

We performed a simulation of a ${ }^{252} \mathrm{Cf}$ source in the middle of the liquid scintillator array shown in Fig. 1. The reconstruction of the source neutron spectrum is shown in Fig. 13. The estimated average source neutron energy is $3.05 \mathrm{MeV}$, but this value cannot be compared to the true average energy of ${ }^{252} \mathrm{Cf}$ spontaneous fission neutrons, because it is computed over the truncated energy distribution shown in Fig. 13.
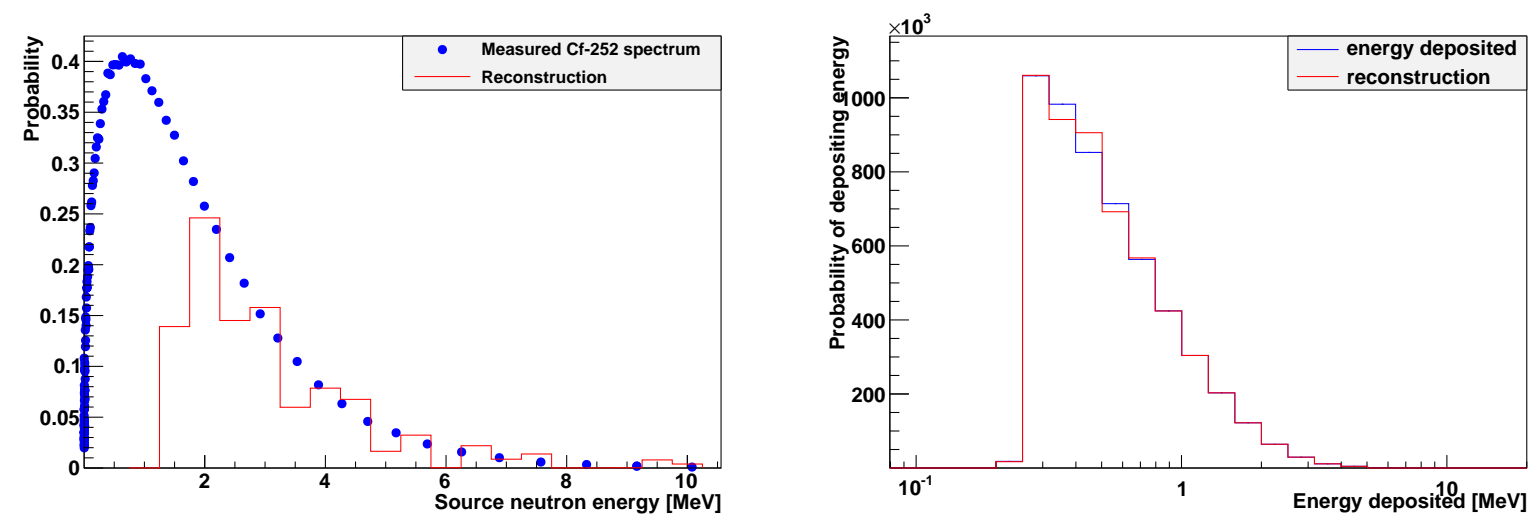

Figure 13: (a) Reconstruction of the source neutron energy spectrum using Eq. 28 (red), along with the measured ${ }^{252} \mathrm{Cf}$ spectrum from Ref. [1]. (b) Reconstructed spectrum $g^{\text {est }}\left(E_{d}\right)$ of energies deposited by the neutrons, evaluated from Eq. 30. The data is from a MCNPX simulation of a ${ }^{252} \mathrm{Cf}$ source. 


\section{Prediction of multiple scattering correction}

Using equations similar to Eq. 29, we can estimate the fraction of double scattering $f_{2}$ and triple scattering $f_{3}$ by weighing the fractions $f_{2}\left(E_{i}\right)$ and $f_{3}\left(E_{i}\right)$ at different source energies $E_{i}$ by $w_{E_{i}}$ (given by Eq. 27 when Eq. 28 is minimized):

$$
\begin{aligned}
& f_{2}=\sum_{i=1}^{19} w_{E_{i}} f_{2}\left(E_{i}\right) \\
& f_{3}=\sum_{i=1}^{19} w_{E_{i}} f_{3}\left(E_{i}\right)
\end{aligned}
$$

The functions $f_{2}\left(E_{i}\right)$ and $f_{3}\left(E_{i}\right)$ are the curves shown in Fig. 8 .

For the 3 mono-energetic neutron sources of section 4.1 , and the ${ }^{252} \mathrm{Cf}$ source of section 4.2 , the estimated fractions $f_{2}$ and $f_{3}$ are given in table 1 . 


\section{Mass correction using multiple scattering correction}

A simulation was run to see if the strength of a ${ }^{252} \mathrm{Cf}$ could be more accurately determined using the multiple scattering correction. For this simulation, we used the same geometry as for the previous cases. The count distribution $b_{n}$, along with the first 3 moments are shown in Fig. 14.
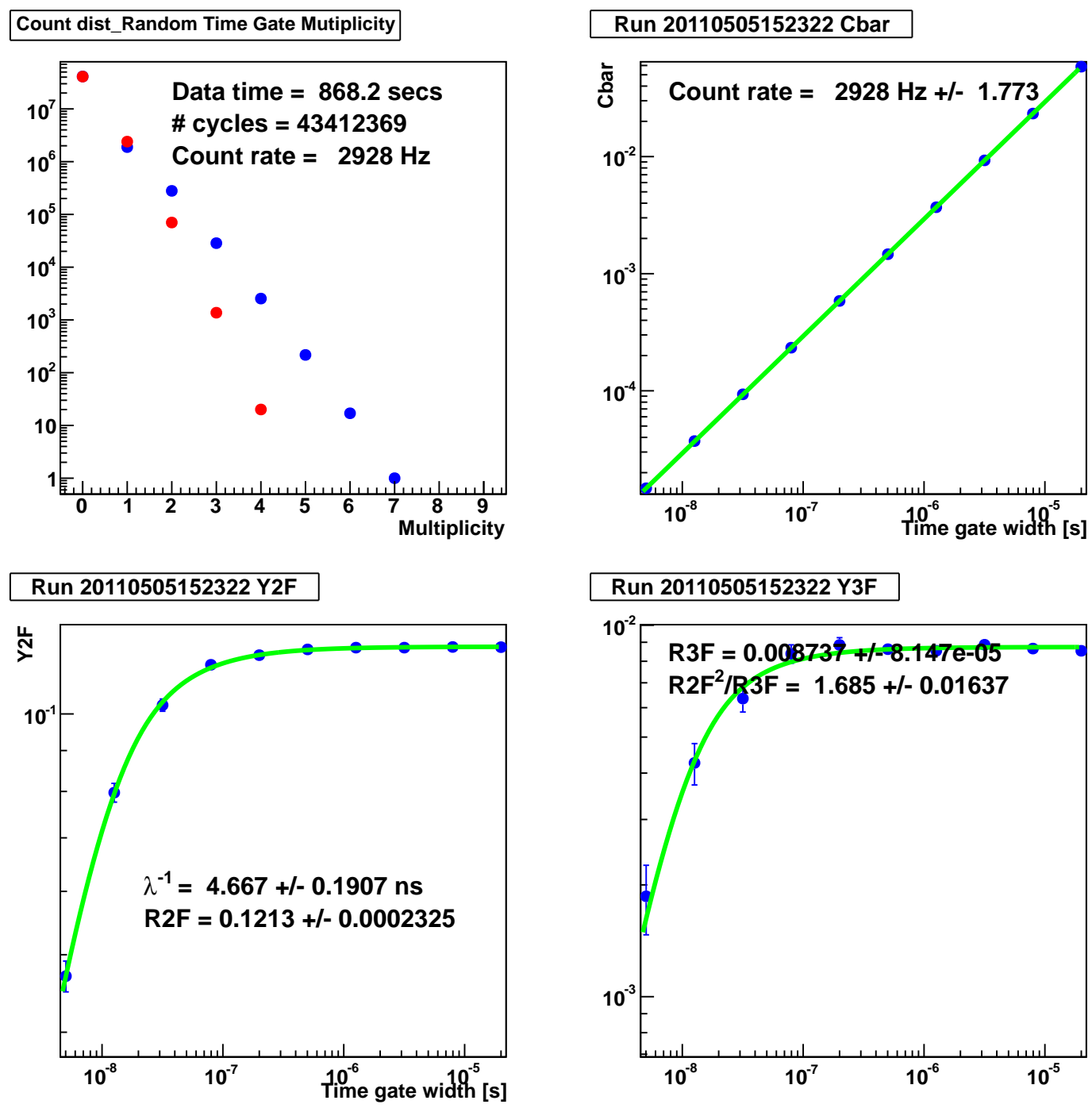

Figure 14: Count distribution $b_{n}(T=20 \mu s)$ and first three moments $\bar{C}(T), Y_{2}(T)$ and $Y_{3}(T)$ as a function of time gate width $T$. The moments are fit using Eqs. 1, 5 and 8, with $\alpha$ set to 0. The data are from an MCNPX simulation of a ${ }^{252} \mathrm{Cf}$ source in the middle of the liquid scintillator array shown in Fig. 1.

For ${ }^{252} \mathrm{Cf}$, the multiplication $M$ is 1 and the parameter $\alpha$ is 0 . If one tries to solve for the mass of ${ }^{252} \mathrm{Cf}$ using this simulated data set, the system of equations 15 only has two unknowns $F_{s}$ and $\varepsilon$ and the first 2 
moments

$$
\left\{\begin{array}{l}
R_{1}=\varepsilon \bar{v}_{s p} F_{s} \\
R_{2 F}=\varepsilon D_{2 s}
\end{array}\right.
$$

contain enough information to solve for the efficiency $\varepsilon$ and the source strength $\bar{v}_{s p} F_{s}$. Knowing that $D_{2 s}$ is equal to 1.595975 for ${ }^{252} \mathrm{Cf}$, the detection efficiency $\varepsilon$ is equal to $7.6 \%$ with a relative error of $0.2 \%$ and the ${ }^{252} \mathrm{Cf}$ source strength is $38,524 \pm 0.2 \%$ neutrons/s. For this simulation, the input source strength was 39,761 neutrons/s. The source strength is thus underestimated by $3.2 \%$ (that is 16 standard deviations) with the equations with no multiple scattering correction. With $\bar{v}_{s p}$ equal to 3.7727 , one gets a ${ }^{252} \mathrm{Cf}$ mass of $16.46 \pm 0.2 \%$ nanograms.

If we account for multiple scattering, we will use the following system of equations instead of Eqs. 33:

$$
\left\{\begin{array}{l}
R_{1}=\left(1+f_{2}+2 f_{3}\right) \varepsilon \bar{v}_{s p} F_{s} \\
R_{2 F}=\frac{f_{2}+3 f_{3}}{1+f_{2}+2 f_{3}}+\left(1+f_{2}+2 f_{3}\right) \varepsilon D_{2 s}
\end{array}\right.
$$

Using the multiple scattering corrections given in table 1, the source strength now becomes 39,925 neutrons/s and the efficiency $7.3 \%$ with a relative error of $0.2 \%$. With the multiple scattering correction, the source strength is within 2 standard deviations from the true value, which is a significant improvement over the estimate not using the multiple scattering correction.

The same checks were done for mono-energetic random (i.e. uncorrelated) neutron sources emitting single neutrons at a time. In these cases, we showed that the measured value of $R_{2 F}$ was equal to the first term in the equation for $R_{2 F}$ in the system of equations 34, which is consistent with $D_{2 s}$ being equal to 0 , which is the correct uncorrelated value. Of course, it is impossible to determine the detection efficiency $\varepsilon$, nor the mass of such sources. 


\section{Conclusion}

With the help of theoretical expressions for the moments of count distributions that account for multiple scatterings of neutrons, we showed that it is possible to determine the fractions of neutrons scattering twice and thrice between liquid scintillator cells from the first 3 moments of measured count distributions. These multiple scattering fractions can be determined for any neutron source, whether monoenergetic or not, and they strongly depend on the energy of the source neutrons.

For nuclear materials undergoing either spontaneous or induced fission, the same theoretical expressions for the moments can be used in conjunction with the measured deposited energy spectrum to apply corrections to the estimates of the detection efficiency, the system multiplication, and the masses of the sources under measurement. A simple simulation using a Californium source showed that the determination of the

${ }^{252} \mathrm{Cf}$ mass was greatly improved using the multiple scattering correction. While the ${ }^{252} \mathrm{Cf}$ uncorrected mass was 16 standard deviations off, the mass was within 2 standard deviations of the correct value with the multiple scattering correction.

Also, we were able to show that measuring the spectrum of fast neutrons depositing energy in the liquid scintillator cells reveals spectral information about the neutron source. 


\section{A Derivation of the moment equations for neutrons scattering multiple times}

One can derive the expressions for $\bar{C}(T)$ starting from the equations for the $\Lambda_{n}(T)$ including single neutrons registering multiple counts in different liquid scintillator cells in the limit of large time gate width $T$ :

$$
\begin{aligned}
& \lim _{T \gg \lambda-1} \frac{\Lambda_{n}(T)}{F_{S} T}=\sum_{v=n}^{\infty} P_{V}\left(\begin{array}{l}
v \\
n
\end{array}\right) \varepsilon^{n}(1-\varepsilon)^{v-n} \alpha_{1}^{n} \\
& +\sum_{v=n-1}^{\infty} P_{v}\left(\begin{array}{c}
v \\
n-1
\end{array}\right) \varepsilon^{n-1}(1-\varepsilon)^{v-n+1}\left(\begin{array}{c}
n-1 \\
1
\end{array}\right) \alpha_{1}^{n-2} \alpha_{2} \\
& +\sum_{v=n-2}^{\infty} P_{v}\left(\begin{array}{c}
v \\
n-2
\end{array}\right) \varepsilon^{n-2}(1-\varepsilon)^{v-n+2}\left(\begin{array}{c}
n-2 \\
2
\end{array}\right) \alpha_{1}^{n-4} \alpha_{2}^{2} \\
& +\sum_{v=n-3}^{\infty} P_{v}\left(\begin{array}{c}
v \\
n-3
\end{array}\right) \varepsilon^{n-3}(1-\varepsilon)^{v-n+3}\left(\begin{array}{c}
n-3 \\
3
\end{array}\right) \alpha_{1}^{n-6} \alpha_{2}^{3} \\
& +\sum_{v=n-4}^{\infty} P_{v}\left(\begin{array}{c}
v \\
n-4
\end{array}\right) \varepsilon^{n-4}(1-\varepsilon)^{v-n+4}\left(\begin{array}{c}
n-4 \\
4
\end{array}\right) \alpha_{1}^{n-8} \alpha_{2}^{4} \\
& +\ldots \\
& +\sum_{v=n-2}^{\infty} P_{v}\left(\begin{array}{c}
v \\
n-2
\end{array}\right) \varepsilon^{n-2}(1-\varepsilon)^{v-n+2}\left(\begin{array}{c}
n-2 \\
1
\end{array}\right) \alpha_{1}^{n-3} \alpha_{3} \\
& +\sum_{v=n-4}^{\infty} P_{v}\left(\begin{array}{c}
v \\
n-4
\end{array}\right) \varepsilon^{n-4}(1-\varepsilon)^{v-n+4}\left(\begin{array}{c}
n-4 \\
2
\end{array}\right) \alpha_{1}^{n-6} \alpha_{3}^{2} \\
& +\sum_{v=n-6}^{\infty} P_{v}\left(\begin{array}{c}
v \\
n-6
\end{array}\right) \varepsilon^{n-6}(1-\varepsilon)^{v-n+6}\left(\begin{array}{c}
n-6 \\
3
\end{array}\right) \alpha_{1}^{n-9} \alpha_{3}^{3} \\
& +\sum_{v=n-8}^{\infty} P_{v}\left(\begin{array}{c}
v \\
n-8
\end{array}\right) \varepsilon^{n-8}(1-\varepsilon)^{v-n+8}\left(\begin{array}{c}
n-8 \\
4
\end{array}\right) \alpha_{1}^{n-12} \alpha_{3}^{4} \\
& +\ldots \\
& +\sum_{v=n-3}^{\infty} P_{v}\left(\begin{array}{c}
v \\
n-3
\end{array}\right) \varepsilon^{n-3}(1-\varepsilon)^{v-n+3} \frac{(n-3) !}{1 ! 1 !(n-5) !} \alpha_{1}^{n-5} \alpha_{2} \alpha_{3} \\
& +\sum_{v=n-4}^{\infty} P_{v}\left(\begin{array}{c}
v \\
n-4
\end{array}\right) \varepsilon^{n-4}(1-\varepsilon)^{v-n+4} \frac{(n-4) !}{2 ! 1 !(n-7) !} \alpha_{1}^{n-7} \alpha_{2}^{2} \alpha_{3} \\
& +\sum_{v=n-5}^{\infty} P_{v}\left(\begin{array}{c}
v \\
n-5
\end{array}\right) \varepsilon^{n-5}(1-\varepsilon)^{v-n+5} \frac{(n-5) !}{3 ! 1 !(n-9) !} \alpha_{1}^{n-9} \alpha_{2}^{3} \alpha_{3} \\
& +\sum_{v=n-5}^{\infty} P_{v}\left(\begin{array}{c}
v \\
n-5
\end{array}\right) \varepsilon^{n-5}(1-\varepsilon)^{v-n+5} \frac{n-5}{3 ! 1 !(n-9) !} \alpha_{1}^{n-9} \alpha_{2}^{3} \alpha_{3} \\
& +\ldots \\
& +\sum_{v=n-5}^{\infty} P_{v}\left(\begin{array}{c}
v \\
n-5
\end{array}\right) \varepsilon^{n-5}(1-\varepsilon)^{v-n+5} \frac{(n-5) !}{1 ! 2 !(n-8) !} \alpha_{1}^{n-8} \alpha_{2} \alpha_{3}^{2} \\
& +\sum_{v=n-6}^{\infty} P_{v}\left(\begin{array}{c}
v \\
n-6
\end{array}\right) \varepsilon^{n-6}(1-\varepsilon)^{v-n+6} \frac{(n-6) !}{2 ! 2 !(n-10) !} \alpha_{1}^{n-10} \alpha_{2}^{2} \alpha_{3}^{2} \\
& +\ldots
\end{aligned}
$$


where $\Lambda_{n}(T)$ is the probability of measuring $n$ neutrons from a fission chain originated by a single spontaneous fission in a time gate of width $T . \alpha_{2}$ and $\alpha_{3}$ are the probabilities that a neutron detected in one liquid scintillator cell scatter and registers one or two more counts in other liquid scintillator cells, respectively. $\alpha_{1}$ is the probability that a neutron detected in one liquid scintillator cells does not register more counts in other liquid scintillator cells, so that the $\alpha$ distribution is normalized, $\alpha_{1}=1-\alpha_{2}-\alpha_{3}$. If we were to account for higher order scattering events, we would have $\alpha_{1}=1-\sum_{i=2}^{\infty} \alpha_{i}$.

Here is how to interpret Eq. 35: the first term in the series is a summation of probabilities to detect $n$ neutrons from a single fission chain producing $v$ neutrons where none of the $v$ neutrons registered multiple counts. Each one of the probabilities in the sum is thus multiplied by $\alpha_{1}^{n}$, the probability that none of the $n$ neutrons detected registered multiple counts. The second term in the series is a summation of probabilities to detect $n$ neutrons from a single fission chain producing $v$ neutrons, where $n-2$ of the $v$ neutrons produced by the chain did not register multiple counts in liquid scintillator cells, and one neutron from this fission chain registered two counts in different liquid scintillator cells. Each one of the probabilities in the sum is thus multiplied by $\alpha_{1}^{n-2}$ and $\alpha_{2}$, the probability that $n-2$ neutrons registered single detected events and the probability that one neutron was detected in two different cells. Because the neutron registering two counts can be any of the $n-1$ neutrons, we need to multiply these probabilities by the number of ways one can choose 1 neutron among $n-1$ neutrons, i.e. $\left(\begin{array}{c}n-1 \\ 1\end{array}\right)$.

The first term in the second group of terms in Eq. 35 is a summation of probabilities to detect $n$ neutrons from a single fission chain producing $v$ neutrons, where $n-3$ of the $v$ neutrons produced by the chain did not register multiple counts in liquid scintillator cells, and one neutron from this fission chain registered three counts in different liquid scintillator cells. Each one of the probabilities in the sum is thus multiplied by $\alpha_{1}^{n-3}$ and $\alpha_{3}$, the probability that $n-3$ neutrons registered single detected events and the probability that one neutron was detected in three different cells. Because the neutron registering three counts can be any of the $n-2$ neutrons, we need to multiply these probabilities by the number of ways one can choose 1 neutron among $n-2$ neutrons, i.e. $\left(\begin{array}{c}n-2 \\ 1\end{array}\right)$.

If one denotes by $\Lambda_{n}^{*}(T)$ the expressions $\Lambda_{n}(T)$ when neutrons are not counted multiple times (that is with $\alpha_{i>1}=0$ ), we get

$$
\lim _{T \gg \lambda-1} \Lambda_{n}^{*}(T)=F_{S} T \sum_{v=n}^{\infty} P_{v}\left(\begin{array}{l}
v \\
n
\end{array}\right) \varepsilon^{n}(1-\varepsilon)^{v-n}
$$

One notices that each one of the terms in the series in Eq. 35 is a different order of $\Lambda_{n}^{*}(T)$ multiplied by a multinomial coefficient of the form

$$
\frac{\left(\sum_{i=1}^{\infty} j_{i}\right) !}{\prod_{i=1}^{\infty} j_{i} !} \prod_{i=1}^{\infty} \alpha_{i}^{j_{i}}
$$

The multinomial coefficients are counting the number of ways to distribute the different populations of neutrons (those registering one count, those registering two counts, etc.).

When there are only two kinds of multiple counts (e.g. $\alpha_{1}$ and $\alpha_{2}$ ), the multinomial reduces to a binomial coefficient

$$
\left(\begin{array}{c}
i+j \\
i
\end{array}\right) \alpha_{m}^{i} \alpha_{n}^{j}
$$

Using the $\Lambda_{n}^{*}(T)$ notation, the first term of Eq. 35 becomes $\alpha_{1}^{n} \Lambda_{n}^{*}$, the second term $\left(\begin{array}{c}n-1 \\ 1\end{array}\right) \alpha_{1}^{n-2} \alpha_{2} \Lambda_{n-1}^{*}$, the 
third one $\left(\begin{array}{c}n-2 \\ 2\end{array}\right) \alpha_{1}^{n-4} \alpha_{2}^{2} \Lambda_{n-2}^{*}$, etc. Eq. 35 can thus be rewritten more compactly as

$$
\begin{aligned}
\lim _{T \gg \lambda^{-1}} \Lambda_{n}(T) & \\
& \alpha_{1}^{n} \Lambda_{n}^{*}+\left(\begin{array}{c}
n-1 \\
1
\end{array}\right) \alpha_{1}^{n-2} \alpha_{2} \Lambda_{n-1}^{*}+\left(\begin{array}{c}
n-2 \\
2
\end{array}\right) \alpha_{1}^{n-4} \alpha_{2}^{2} \Lambda_{n-2}^{*}+\left(\begin{array}{c}
n-3 \\
3
\end{array}\right) \alpha_{1}^{n-6} \alpha_{2}^{3} \Lambda_{n-3}^{*}+\left(\begin{array}{c}
n-4 \\
4
\end{array}\right) \alpha_{1}^{n-8} \alpha_{2}^{4} \Lambda_{n-4}^{*}+\ldots \\
& +\left(\begin{array}{c}
n-2 \\
1
\end{array}\right) \alpha_{1}^{n-3} \alpha_{3} \Lambda_{n-2}^{*}+\left(\begin{array}{c}
n-4 \\
2
\end{array}\right) \alpha_{1}^{n-6} \alpha_{3}^{2} \Lambda_{n-4}^{*}+\left(\begin{array}{c}
n-6 \\
3
\end{array}\right) \alpha_{1}^{n-9} \alpha_{3}^{3} \Lambda_{n-6}^{*}+\left(\begin{array}{c}
n-8 \\
4
\end{array}\right) \alpha_{1}^{n-12} \alpha_{3}^{4} \Lambda_{n-8}^{*}+\ldots \\
& +\frac{(n-3) !}{1 ! 1 !(n-5) !} \alpha_{1}^{n-5} \alpha_{2} \alpha_{3} \Lambda_{n-3}^{*}+\frac{(n-4) !}{2 ! 1 !(n-7) !} \alpha_{1}^{n-7} \alpha_{2}^{2} \alpha_{3} \Lambda_{n-4}^{*}+\frac{(n-5) !}{3 ! 1 !(n-9) !} \alpha_{1}^{n-9} \alpha_{2}^{3} \alpha_{3} \Lambda_{n-5}^{*}+\ldots \\
& +\frac{(n-5) !}{1 ! 2 !(n-8) !} \alpha_{1}^{n-8} \alpha_{2} \alpha_{3}^{2} \Lambda_{n-5}^{*}+\frac{(n-6) !}{2 ! 2 !(n-10) !} \alpha_{1}^{n-10} \alpha_{2}^{2} \alpha_{3}^{2} \Lambda_{n-6}^{*}+\ldots
\end{aligned}
$$

or

$$
\begin{aligned}
\lim _{T \gg \lambda^{-1}} \Lambda_{n}(T) & =\sum_{i=0}^{i \leq n / 2}\left(\begin{array}{c}
n-i \\
i
\end{array}\right) \alpha_{1}^{n-2 i} \alpha_{2}^{i} \Lambda_{n-i}^{*} \\
& +\sum_{j=1}^{j \leq n / 3}\left(\begin{array}{c}
n-2 j \\
j
\end{array}\right) \alpha_{1}^{n-3 j} \alpha_{3}^{j} \Lambda_{n-2 j}^{*} \\
& +\sum_{i=1}^{i \leq(n-3) / 2} \frac{(n-2-i) !}{i ! 1 !(n-3-2 i) !} \alpha_{1}^{n-3-2 i} \alpha_{2}^{i} \alpha_{3} \Lambda_{n-2-i}^{*} \\
& +\sum_{i=1}^{i \leq(n-6) / 2} \frac{(n-4-i) !}{i ! 2 !(n-6-2 i) !} \alpha_{1}^{n-6-2 i} \alpha_{2}^{i} \alpha_{3}^{2} \Lambda_{n-4-i}^{*} \\
& +\ldots
\end{aligned}
$$

which can be rewritten as

$$
\lim _{T \gg \lambda^{-1}} \Lambda_{n}(T)=\sum_{j=0}^{j \leq n / 3} \sum_{i=0}^{i \leq(n-3 * j) / 2} \frac{(n-2 j-i) !}{i ! j !(n-3 j-2 i) !} \alpha_{1}^{n-3 j-2 i} \alpha_{2}^{i} \alpha_{3}^{j} \Lambda_{n-2 j-i}^{*}
$$

The first few $\Lambda_{n}(T)$ are thus

$$
\begin{aligned}
& \lim _{T \gg \lambda^{-1}} \Lambda_{1}(T)=\Lambda_{1}^{*} \alpha_{1} \\
& \lim _{T \gg \lambda^{-1}} \Lambda_{2}(T)=\Lambda_{2}^{*} \alpha_{1}^{2}+\Lambda_{1}^{*} \alpha_{2} \\
& \lim _{T \gg \lambda^{-1}} \Lambda_{3}(T)=\Lambda_{3}^{*} \alpha_{1}^{3}+\Lambda_{2}^{*}\left(\begin{array}{l}
2 \\
1
\end{array}\right) \alpha_{1} \alpha_{2}+\Lambda_{1}^{*} \alpha_{3} \\
& \lim _{T \gg \lambda^{-1}} \Lambda_{4}(T)=\Lambda_{4}^{*} \alpha_{1}^{4}+\Lambda_{3}^{*}\left(\begin{array}{l}
3 \\
1
\end{array}\right) \alpha_{1}^{2} \alpha_{2}+\Lambda_{2}^{*} \alpha_{2}^{2}+\Lambda_{2}^{*}\left(\begin{array}{l}
2 \\
1
\end{array}\right) \alpha_{1} \alpha_{3} \\
& \lim _{T \gg \lambda^{-1}} \Lambda_{5}(T)=\Lambda_{5}^{*} \alpha_{1}^{5}+\Lambda_{4}^{*}\left(\begin{array}{l}
4 \\
1
\end{array}\right) \alpha_{1}^{3} \alpha_{2}+\Lambda_{3}^{*}\left(\begin{array}{l}
3 \\
2
\end{array}\right) \alpha_{1} \alpha_{2}^{2}+\Lambda_{3}^{*}\left(\begin{array}{l}
3 \\
1
\end{array}\right) \alpha_{1}^{2} \alpha_{3}+\Lambda_{2}^{*} \frac{2 !}{1 ! 1 ! 0 !} \alpha_{2} \alpha_{3} \\
& \lim _{T \gg \lambda^{-1}} \Lambda_{6}(T)=\Lambda_{6}^{*} \alpha_{1}^{6}+\Lambda_{5}^{*}\left(\begin{array}{l}
5 \\
1
\end{array}\right) \alpha_{1}^{4} \alpha_{2}+\Lambda_{4}^{*}\left(\begin{array}{l}
4 \\
2
\end{array}\right) \alpha_{1}^{2} \alpha_{2}^{2}+\Lambda_{3}^{*} \alpha_{2}^{3}+\Lambda_{4}^{*}\left(\begin{array}{l}
4 \\
1
\end{array}\right) \alpha_{1}^{3} \alpha_{3}+\Lambda_{2}^{*} \alpha_{3}^{2}+\Lambda_{3}^{*} \frac{3 !}{1 ! 1 ! 1 !} \alpha_{1} \alpha_{2} \alpha_{3}
\end{aligned}
$$


Using these expressions for $\Lambda_{n}(T)$, the measured count rate $\bar{C}(T)$ can be written as

$$
\begin{aligned}
\bar{C}(T) & =\sum_{i=1}^{\infty}\left(\begin{array}{l}
i \\
1
\end{array}\right) \Lambda_{i} \\
& =\Lambda_{1}+2 \Lambda_{2}+3 \Lambda_{3}+4 \Lambda_{4}+5 \Lambda_{5}+6 \Lambda_{6}+7 \Lambda_{7}+8 \Lambda_{8}+9 \Lambda_{9}+10 \Lambda_{10}+11 \Lambda_{11}+12 \Lambda_{12}+\ldots \\
& =\left(1+\alpha_{2}+2 \alpha_{3}\right) \Lambda_{1}^{*}+2\left(1+\alpha_{2}+2 \alpha_{3}\right) \Lambda_{2}^{*}+3\left(1+\alpha_{2}+2 \alpha_{3}\right) \Lambda_{3}^{*}+4\left(1+\alpha_{2}+2 \alpha_{3}\right) \Lambda_{4}^{*}+\ldots \\
& =\left(1+\alpha_{2}+2 \alpha_{3}\right)\left(\Lambda_{1}^{*}+2 \Lambda_{2}^{*}+3 \Lambda_{3}^{*}+4 \Lambda_{4}^{*}+\ldots\right) \\
& =\left(1+\alpha_{2}+2 \alpha_{3}\right) \sum_{i=1}^{\infty}\left(\begin{array}{l}
i \\
1
\end{array}\right) \Lambda_{i}^{*} \\
& =\left(1+\alpha_{2}+2 \alpha_{3}\right) \bar{C}^{*}(T)
\end{aligned}
$$

which means that the true count rate $\bar{C}^{*}(T)$ is increased by the multiplier $1+\alpha_{2}+2 \alpha_{3}$. In the limiting case where $\alpha_{2}$ is 1 , that is, all neutrons register two counts in the liquid scintillators, the measured count rate is twice the true count rate. In the limiting case where $\alpha_{3}$ is 1 , where all neutrons register three counts in the liquid scintillators, the measured count rate is thrice the true count rate. These two limiting cases are consistent with our expectations.

Similarly, the moment $Y_{2}(T)$ can be written as

$$
\begin{aligned}
Y_{2}(T) & =\sum_{i=2}^{\infty}\left(\begin{array}{l}
i \\
2
\end{array}\right) \Lambda_{i} \\
& =\left(\alpha_{2}+3 \alpha_{3}\right) \sum_{i=1}^{\infty}\left(\begin{array}{l}
i \\
1
\end{array}\right) \Lambda_{i}^{*}+\left(1+\alpha_{2}^{2}+4 \alpha_{3}^{2}+4 \alpha_{2} \alpha_{3}+2 \alpha_{2}+4 \alpha_{3}\right) \sum_{i=2}^{\infty}\left(\begin{array}{l}
i \\
2
\end{array}\right) \Lambda_{i}^{*} \\
& =\left(\alpha_{2}+3 \alpha_{3}\right) \bar{C}^{*}(T)+\left(1+\alpha_{2}^{2}+4 \alpha_{3}^{2}+4 \alpha_{2} \alpha_{3}+2 \alpha_{2}+4 \alpha_{3}\right) Y_{2}^{*}(T) \\
& =\left(\alpha_{2}+3 \alpha_{3}\right) \bar{C}^{*}(T)+\left(1+\alpha_{2}+2 \alpha_{3}\right)^{2} Y_{2}^{*}(T)
\end{aligned}
$$

and $Y_{3}(T)$ as

$$
\begin{aligned}
Y_{3}(T) & =\sum_{i=3}^{\infty}\left(\begin{array}{l}
i \\
3
\end{array}\right) \Lambda_{i} \\
& =\alpha_{3} \sum_{i=1}^{\infty}\left(\begin{array}{l}
i \\
1
\end{array}\right) \Lambda_{i}^{*}+2\left(\alpha_{2}+\alpha_{2}^{2}+3 \alpha_{3}+5 \alpha_{2} \alpha_{3}+6 \alpha_{3}^{2}\right) \sum_{i=2}^{\infty}\left(\begin{array}{l}
i \\
2
\end{array}\right) \Lambda_{i}^{*} \\
& +\left(1+3 \alpha_{2}+3 \alpha_{2}^{2}+\alpha_{2}^{3}+6 \alpha_{3}+12 \alpha_{2} \alpha_{3}+6 \alpha_{2}^{2} \alpha_{3}+12 \alpha_{3}^{2}+12 \alpha_{2} \alpha_{3}^{2}+8 \alpha_{3}^{3}\right) \sum_{i=3}^{\infty}\left(\begin{array}{l}
i \\
3
\end{array}\right) \Lambda_{i}^{*} \\
& =\alpha_{3} \bar{C}^{*}(T)+2\left(1+\alpha_{2}+2 \alpha_{3}\right)\left(\alpha_{2}+3 \alpha_{3}\right) Y_{2}^{*}(T)+\left(1+\alpha_{2}+2 \alpha_{3}\right)^{3} Y_{3}^{*}(T)
\end{aligned}
$$

Dividing both expressions by $\bar{C}(T)$, we get

$$
Y_{2 F}(T)=\frac{\alpha_{2}+3 \alpha_{3}}{1+\alpha_{2}+2 \alpha_{3}}+\left(1+\alpha_{2}+2 \alpha_{3}\right) Y_{2 F}^{*}(T)
$$

and

$$
Y_{3 F}(T)=\frac{\alpha_{3}}{1+\alpha_{2}+2 \alpha_{3}}+2\left(\alpha_{2}+3 \alpha_{3}\right) Y_{2 F}^{*}(T)+\left(1+\alpha_{2}+2 \alpha_{3}\right)^{2} Y_{3 F}^{*}(T)
$$


An alternative way to get to the same result is by considering the generating function for the $e_{n}(\varepsilon)$ distribution, which gives the probability of detecting $n$ neutrons from a single fission chain. In the case when neutrons never multiple scatter between liquid scintillator cells, the generating function for the $e_{n}(\varepsilon)$ distribution can be written as

$$
h(y)=\sum_{v=0}^{\infty} e_{n}(\varepsilon) y^{n}
$$

When neutrons are allowed to multiple scatter between liquid scintillator cells, the variable $y$ can be replaced by the following polynomial in $y$

$$
f(y) \rightarrow(1-\varepsilon)+\varepsilon\left(1-\alpha_{2}-\alpha_{3}\right) y+\varepsilon \alpha_{2} y^{2}+\varepsilon \alpha_{3} y^{3}
$$

where the first term $(1-\varepsilon)$ - which could also be written as $(1-\varepsilon) y^{0}-$ is the probability that a neutron is not detected; the second term (or more precisely the coefficient of y) $\varepsilon\left(1-\alpha_{2}-\alpha_{3}\right)$ is the probability that a neutron is detected by a single liquid scintillator cell; the third polynomial coefficient $\varepsilon \alpha_{2}$ is the probability that a neutron is detected by a liquid scintillator cell, scatters and is detected once and only once more by another liquid scintillator cell; and finally the last polynomial coefficient $\varepsilon \alpha_{3}$ is the probability that a neutron is detected thrice by 3 different liquid scintillator cells. The powers in $y$ count the number of times a single neutron is detected by the array: $0,1,2$ or 3 times.

Taking the first derivative of $h(f(y))$ with respect to $y$, and setting $y$ to 1 , we get

$$
\begin{aligned}
\frac{\partial h}{\partial y} & =h^{\prime} f^{\prime} \\
& =\varepsilon h^{\prime}\left(\left(1-\alpha_{2}-\alpha_{3}\right)+2 \alpha_{2}+3 \alpha_{3}\right) \\
& =\varepsilon h^{\prime}\left(1+\alpha_{2}+2 \alpha_{3}\right) \\
& =R_{1}^{*}\left(1+\alpha_{2}+2 \alpha_{3}\right)
\end{aligned}
$$

The second derivative leads to

$$
\begin{aligned}
\frac{1}{2 !} \frac{\partial^{2} h}{\partial y^{2}} & =\frac{1}{2 !}\left(h^{\prime \prime} f^{\prime 2}+h^{\prime} d^{\prime \prime}\right) \\
& =\frac{1}{2 !} h^{\prime \prime}\left(\varepsilon\left(1-\alpha_{2}-\alpha_{3}\right)+2 \varepsilon \alpha_{2}+3 \varepsilon \alpha_{3}\right)^{2}+h^{\prime} \varepsilon\left(\alpha_{2}+3 \alpha_{3}\right) \\
& =R_{2}^{*}\left(1+\alpha_{2}+2 \alpha_{3}\right)^{2}+R_{1}^{*}\left(\alpha_{2}+3 \alpha_{3}\right)
\end{aligned}
$$

Similarly, the third derivative is written as

$$
\begin{aligned}
\frac{1}{3 !} \frac{\partial^{3} h}{\partial y^{3}} & =\frac{1}{3 !}\left(h^{\prime \prime \prime} f^{\prime 3}+3 h^{\prime \prime} f^{\prime} f^{\prime \prime}+h^{\prime} d^{\prime \prime \prime}\right) \\
& =\frac{1}{3 !} h^{\prime \prime \prime} \varepsilon^{3}\left(1+\alpha_{2}+2 \alpha_{3}\right)^{3}+h^{\prime \prime} \varepsilon^{2}\left(1+\alpha_{2}+2 \alpha_{3}\right)\left(\alpha_{2}+3 \alpha_{3}\right)+h^{\prime} \varepsilon \alpha_{3} \\
& =\left(1+\alpha_{2}+2 \alpha_{3}\right)^{3} R_{3}^{*}+2\left(1+\alpha_{2}+2 \alpha_{3}\right)\left(\alpha_{2}+3 \alpha_{3}\right) R_{2}^{*}+\alpha_{3} R_{1}^{*}
\end{aligned}
$$

Dividing Eqs. 51 and 52 by Eq. 50, we get the following 2 equations for $R_{2 F}$ and $R_{3 F}$ :

$$
R_{2 F}=\frac{\alpha_{2}+3 \alpha_{3}}{1+\alpha_{2}+2 \alpha_{3}}+\left(1+\alpha_{2}+2 \alpha_{3}\right) R_{2 F}^{*}
$$




$$
R_{3 F}=\frac{\alpha_{3}}{1+\alpha_{2}+2 \alpha_{3}}+2\left(\alpha_{2}+3 \alpha_{3}\right) R_{2 F}^{*}+\left(1+\alpha_{2}+2 \alpha_{3}\right)^{2} R_{3 F}^{*}
$$

\section{References}

[1] "Simulation of Neutron and Gamma Ray Emission from Fission and Photofission," UCRL-AR228518, Lawrence Livermore National Laboratory (2010). 